\title{
Odd-parity multipole fluctuation and unconventional superconductivity in locally noncentrosymmetric crystal
}

\author{
Jun Ishizuka ${ }^{1, *}$ and Youichi Yanase ${ }^{1}$ \\ ${ }^{1}$ Department of Physics, Graduate School of Science, Kyoto University, Kyoto 606-8502, Japan
}

(Dated: December 19, 2018)

\begin{abstract}
A microscopic calculation and symmetry argument reveal superconductivity in the vicinity of parity-violating magnetic order. In a crystal structure lacking local space inversion symmetry, an augmented cluster magnetic multipole order may break global inversion symmetry, and therefore it is classified into an odd-parity multipole order. We investigate unconventional superconductivity induced by an odd-parity magnetic multipole fluctuation in a two-dimensional two-sublattice Hubbard model motivated by $\mathrm{Sr}_{2} \mathrm{IrO}_{4}$. We find that even-parity superconductivity is more significantly suppressed by spin-orbit coupling than that in a globally noncentrosymmetric system. Consequently, two odd-parity superconducting states are stabilized by magnetic multipole fluctuations in a large spin-orbit coupling region. Both of them are identified as $Z_{2}$ topological superconducting states. The obtained gap function of inter-sublattice pairing shows a gapped or nodal structure protected by nonsymmorphic symmetry. Our finding implies a family of odd-parity topological superconductors. Candidate materials are discussed.
\end{abstract}

\section{INTRODUCTION}

Recent intensive research has clarified the intriguing effects of spin-orbit coupling in locally noncentrosymmetric (NCS) crystals [1-8]. The locally NCS crystal preserves global inversion symmetry in the crystal structure, although inversion symmetry on a local atomic site is lacking. Sublatticedependent antisymmetric spin-orbit coupling (ASOC) appears in locally NCS crystals, and it may induce exotic superconductivity distinct from well-studied globally NCS superconductivity [9-24]. For instance, singlet-triplet mixing [3], a topological crystalline superconductivity of a pair density wave state $[5,7,8]$, and an antiferromagnetic (AFM) Edelstein effect [25-28] have been studied. Motivated by these works, we investigate exotic superconductivity induced by magnetic fluctuation in locally NCS crystals. From the viewpoint of materials, many unconventional superconductors in the vicinity of the AFM critical point, such as iron-based superconductors $[29,30]$ and $\mathrm{CeCoIn}_{5}$ superlattices [2, 31], are classified into locally NCS superconductors. Thus, it is interesting to clarify the effects of local parity violation on AFM-fluctuationinduced superconductivity from a microscopic point of view.

Another topic of recent interest in locally NCS crystals is an odd-parity electromagnetic multipole order [25, 28, 3243], which spontaneously breaks global inversion symmetry through an anisotropic spin and charge distribution. Although previous studies provided a profound understanding of evenparity multipole order in strongly correlated electron systems [44-47], it has been recently recognized that odd-parity electromagnetic multipole order is ubiquitous in materials. For instance, $\mathrm{BaMn}_{2} \mathrm{As}_{2}$ [28, 48], $\mathrm{Sr}_{2} \mathrm{IrO}_{4}$ [39, 40, 49-62], $\mathrm{Cd}_{2} \mathrm{ReO}_{7}$ [33, 63-71] , and $\mathrm{SrTiO}_{3}$ [72-74] have been studied from the viewpoint of odd-parity multipole order. More recently, more than 110 AFM compounds have been identified as odd-parity magnetic multipole states by a group-theoretical analysis [75]. For those compounds, a multipole moment in

* ishizuka.jun.8c@kyoto-u.ac.jp the unit cell (augmented cluster multipole) has an odd parity and leads to parity violation.

Superconductivity near the odd-parity electromagnetic multipole order invokes an unconventional pairing mechanism induced by an odd-parity multipole fluctuation. However, theoretical studies based on microscopic models have not been conducted except for a few works on electric multipole fluctuation [76-78]. Because the AFM order in locally NCS crystals with sublattice-dependent ASOC realizes odd-parity magnetic multipole order [75], our study of fluctuation-induced superconductivity naturally reveals superconductivity due to the magnetic odd-parity multipole fluctuation. The pairing interaction and the resulting superconducting state may be different from those of conventional magnetic-fluctuation-induced superconductivity. Therefore, a different platform of topological superconductivity may be found in this study.

Previous theories based on the random phase approximation (RPA) have investigated the superconductivity induced by AFM fluctuation in globally NCS crystals [15-18, 79]. In this paper, we clarify a peculiar superconducting state and magnetic multipole fluctuation in a locally NCS crystal with the same approximation. To be specific, we analyze a twosublattice Hubbard model with sublattice-dependent ASOC. The crystallographic point group is centrosymmetric $D_{4 h}$ and the local site symmetry is $D_{2 d}$ lacking inversion symmetry. This is a minimal model taking account of the locally NCS structure, spin-orbit coupling, and odd-parity magnetic multipole fluctuation. For instance, $\mathrm{BaMn}_{2} \mathrm{As}_{2}$ and $\mathrm{Sr}_{2} \mathrm{IrO}_{4}$ are captured by this model from the viewpoint of symmetry.

The seemingly conventional $G$-type AFM order in our model shows unbroken translation symmetry, because of the two-sublattice structure peculiar to locally NCS crystals. The magnetic propagation vector is indeed $\boldsymbol{q}=\mathbf{0}$. Instead of the translation symmetry, the space inversion symmetry is broken. Therefore, the AFM order is regarded as an odd-parity magnetic order. From the group-theoretical study [28], the magnetoelectric multipole moment has been classified based on the point group $D_{4 h}$. The magnetic multipole order in the AFM state with $\boldsymbol{m} \| c$ belongs to the $B_{2 u}$ irreducible representation (IR). The candidates of the order parameter are identified as 
the magnetic quadrupole moment and the hexadecapole moment. On the other hand, the $G$-type AFM order with $\boldsymbol{m} \perp c$ corresponds to the magnetic quadrupole and toroidal order. Our calculation takes into account all these magnetic multipole fluctuations. In this paper, we will perform a microscopic study of unconventional superconductivity induced by odd-parity magnetic fluctuation.

This paper is constructed as follows. In Secs. II and III, symmetry operations for pair amplitudes with sublattice degrees of freedom are revealed. We clarify the symmetry properties in the present crystal structure. Since our model preserves nonsymmorphic crystal symmetry, the pair amplitudes have peculiar structures. Section IV introduces a twosublattice Hubbard model with spin-orbit coupling and provides the formulation of the microscopic calculation based on the RPA and Eliashberg equation. Numerical results are shown in Secs. V and VI. In Sec. V, we show the odd-parity magnetic fluctuation and its anisotropy. Effects of the ASOC on the magnetic fluctuations are discussed. In Sec. VI, we identify four stable pairing states, which are distinguished by symmetry. Effects of ASOC in locally NCS crystals are compared with those in globally NCS crystals. It is demonstrated that local parity violation prefers odd-parity superconductivity. Therefore, the $Z_{2}$-topological odd-parity superconductivity in the DIII class is stabilized in a large ASOC region. A brief summary and discussion are given in Sec. VII.

\section{SYMMETRY OF SUPERCONDUCTIVITY IN MULTISUBLATTICE SYSTEMS}

For the classification of pair amplitudes in multicomponent superconductors, we need to take into account the internal degrees of freedom of electrons, which were neglected in classical theories summarized by Sigrist and Ueda [80]. For instance, multiorbital systems have been analyzed in Ref. 81 . We here classify the systems with sublattice degrees of freedom.

To study locally NCS superconductors, it is important to clarify the intersublattice and intrasublattice pairing amplitudes. A complete classification is given by introducing the permutation of sites. We study a single-orbital model for simplicity. An extension to multiorbital and multisublattice systems is straightforward by considering the permutation of local orbitals.

A creation operator of a Bloch state $c_{\boldsymbol{k} m s}^{\dagger}$ with spin $s$ on sublattice $m$ is transformed by a space-group operation,

$$
\begin{aligned}
g c_{\boldsymbol{k} m s}^{\dagger} g^{-1} & =\sum_{\boldsymbol{R}} g c_{s}^{\dagger}\left(\boldsymbol{R}+\boldsymbol{r}_{m}\right) g^{-1} e^{-i \boldsymbol{k} \cdot \boldsymbol{R}} \\
& =\sum_{\boldsymbol{R} s^{\prime}} c_{s^{\prime}}^{\dagger}\left(p\left(\boldsymbol{R}+\boldsymbol{r}_{m}\right)+\boldsymbol{a}\right) D_{s^{\prime} s}^{(1 / 2)}(p) e^{-i \boldsymbol{k} \cdot \boldsymbol{R}} \\
& =e^{i p \boldsymbol{k} \boldsymbol{a}} \sum_{s^{\prime}} c_{p \boldsymbol{k} g m s^{\prime}}^{\dagger} D_{s^{\prime} s}^{(1 / 2)}(p) e^{-i p \boldsymbol{k}\left(\boldsymbol{r}_{g m}-p \boldsymbol{r}_{m}\right)} .
\end{aligned}
$$

Here, $\boldsymbol{R}$ is a basic lattice vector and $\boldsymbol{r}_{m}$ is a relative coordinate of $m$ th sublattice in a unit cell. The operation $g=\{p \mid \boldsymbol{a}\}$ is defined by a conventional Seitz space-group symbol with a point-group operation $p$ and a translation $\boldsymbol{a}$. By choosing a representation matrix indicating the permutation of sites as

$$
D_{m^{\prime} m}^{(\text {perm) }}(g ; \boldsymbol{k})=e^{-i p \boldsymbol{k}\left(\boldsymbol{r}_{g m}-p \boldsymbol{r}_{m}\right)} \delta_{m^{\prime}, g m},
$$

the transformation is simply represented as

$$
g c_{\boldsymbol{k} m s}^{\dagger} g^{-1}=e^{i p \boldsymbol{k} \boldsymbol{a}} \sum_{m^{\prime}, s^{\prime}} c_{p \boldsymbol{k} m^{\prime} s^{\prime}}^{\dagger} D_{s^{\prime} s}^{(1 / 2)}(p) D_{m^{\prime} m}^{(\mathrm{perm})}(g ; \boldsymbol{k}) .
$$

In a superconducting state, a pair amplitude is defined as

$$
F_{m s m^{\prime} s^{\prime}}(\boldsymbol{k})=\left\langle c_{\boldsymbol{k} m s} c_{-\boldsymbol{k} m^{\prime} s^{\prime}}\right\rangle,
$$

where $\langle\cdots\rangle$ denotes the thermal average. The fermion antisymmetry gives

$$
F_{m s m^{\prime} s^{\prime}}(\boldsymbol{k})=-F_{m^{\prime} s^{\prime} m s}(-\boldsymbol{k}) .
$$

From Eqs. (3) and (4), the pair amplitude is translated as

$$
\begin{aligned}
& g F_{m s m^{\prime} s^{\prime}}^{\Gamma_{i}}(\boldsymbol{k}) g^{-1}=\hat{D}(g ; \boldsymbol{k}) \hat{F}^{\Gamma_{i}}(p \boldsymbol{k}) \hat{D}^{\mathrm{T}}(g ; \boldsymbol{k}) \\
& \quad=\sum_{\substack{m_{1}, m_{2} \\
s_{1}, s_{2}}} F_{m_{1} s_{1} m_{2} s_{2}}^{\Gamma_{i}}(p \boldsymbol{k}) \\
& \quad \times D_{s_{1} s_{2} s s^{\prime}}^{(1 / 2)}(p) D_{m_{1} m_{2} m m^{\prime}}^{(\mathrm{perm})}(g ; \boldsymbol{k}) D^{\phi_{\boldsymbol{k}}}\left(g ; \Gamma_{i}\right) .
\end{aligned}
$$

Here, the corresponding representation matrix is

$$
\begin{aligned}
D_{m_{1} m_{2} m m^{\prime}}^{(\mathrm{perm})}(g ; \boldsymbol{k}) & =e^{-i p \boldsymbol{k}\left(\boldsymbol{r}_{g m}-p \boldsymbol{r}_{m}\right)} e^{i p \boldsymbol{k}\left(\boldsymbol{r}_{g m^{\prime}}-p \boldsymbol{r}_{m^{\prime}}\right)} \\
& \times \delta_{m_{1}, g m} \delta_{m_{2}, g m^{\prime}},
\end{aligned}
$$

and $D^{\phi_{\boldsymbol{k}}}\left(g ; \Gamma_{i}\right)$ is the representation matrix of the $\Gamma_{i}$ IR of the gap function, whose characters are explicitly given in Table I for the $D_{4 h}$ point group. Note that $D^{\text {(perm) }}$ is unity for $m=$ $m^{\prime}$.

When the total Hamiltonian commutes with the space inversion operator $I$, the pair amplitude possesses an even parity (odd parity), namely, $I F_{m s m^{\prime} s^{\prime}}^{\Gamma_{g(u)}}(\boldsymbol{k}) I^{-1}=$ $+(-) F_{I m s I m^{\prime} s^{\prime}}^{\Gamma_{g(u)}}(-\boldsymbol{k})$. When the local symmetry on each sublattice has inversion symmetry, $I m=m$, the spin-singlet and spin-triplet pairing states are distinguished by the intrasublattice pair amplitude $I F_{m s m s^{\prime}}^{\Gamma_{g(u)}}(\boldsymbol{k}) I^{-1}=-(+) F_{m s^{\prime} m s}^{\Gamma_{g(u)}}(\boldsymbol{k})$. In the absence of global inversion symmetry, the space inversion parity is not a good quantum number, and parity mixing between the singlet and triplet channels occurs. On the other hand, in locally NCS superconductors the parity mixing appears in a different way. Consequently, the superconducting gap function shows a nontrivial and symmetry-protected structure. We focus on this case in the next section. Hereafter, we assume even-frequency pairing, which is thermodynamically stable [82] in the usual cases.

\section{PAIR AMPLITUDE IN LOCALLY NONCENTROSYMMETRIC CRYSTAL}

For a demonstration, we introduce a typical crystal structure lacking local inversion symmetry and examine the symmetry properties of the pair amplitudes. The example considered throughout this paper is a tetragonal crystal lattice with 
two sublattices, each of which lacks local inversion symmetry. In a crystal structure of $\mathrm{Sr}_{2} \mathrm{IrO}_{4}$, which is depicted in Fig. 1(a), oxygen ions out of the Ir-O layer violate inversion symmetry at an Ir ion. A similar local parity violation appears in iron-based superconductors [Fig. 1(b)], whose zigzag structure of the pnictogen or chalcogen ions also breaks local inversion symmetry, owing to the absence of $\sigma_{v}$ mirror symmetry. Hereafter, we study the crystal structure in Fig. 1(a). A
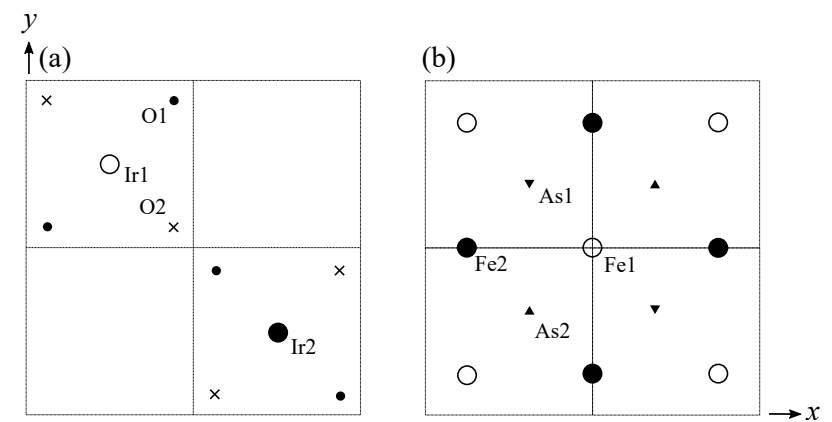

FIG. 1. Crystal structure of (a) $\mathrm{Sr}_{2} \mathrm{IrO}_{4}$ and (b) iron-based superconductors. Two-dimensional Ir and Fe layers with ligands, which break $\sigma_{v}$ mirror symmetry, are plotted. A $D_{2 d}$-type ASOC appears in these structures.

coset decomposition is given by

$$
\begin{aligned}
G & =\{E \mid \mathbf{0}\} T+\{I \mid \mathbf{0}\} T+\left\{2_{z} \mid \boldsymbol{\tau}_{x}+\boldsymbol{\tau}_{y}\right\} T \\
& +\left\{2_{x} \mid \boldsymbol{\tau}_{y}\right\} T+\left\{2_{y} \mid \boldsymbol{\tau}_{x}\right\} T+\left\{4_{z}^{+} \mid \boldsymbol{\tau}_{x}\right\} T+\left\{4_{z}^{-} \mid \boldsymbol{\tau}_{y}\right\} T \\
& +\left\{\sigma_{x} \mid \boldsymbol{\tau}_{y}\right\} T+\left\{\sigma_{y} \mid \boldsymbol{\tau}_{x}\right\} T+\left\{\sigma_{z} \mid \boldsymbol{\tau}_{x}+\boldsymbol{\tau}_{y}\right\} T \\
& +\left\{\sigma_{110} \mid \boldsymbol{\tau}_{x}+\boldsymbol{\tau}_{y}\right\} T+\left\{\sigma_{1-10} \mid \mathbf{0}\right\} T \\
& +\left\{2_{110} \mid \mathbf{0}\right\} T+\left\{2_{1-10} \mid \boldsymbol{\tau}_{\boldsymbol{x}}+\boldsymbol{\tau}_{\boldsymbol{y}}\right\} T \\
& +\left\{I 4_{z}^{+} \mid \boldsymbol{\tau}_{\boldsymbol{x}}\right\} T+\left\{I 4_{z}^{-} \mid \boldsymbol{\tau}_{\boldsymbol{y}}\right\} T
\end{aligned}
$$

where the translation group $T$ defines a Bravais Lattice, and $\boldsymbol{\tau}_{x}=\frac{a}{2} \boldsymbol{e}_{a}, \boldsymbol{\tau}_{y}=\frac{a}{2} \boldsymbol{e}_{b}$ are non-primitive translation vectors.

Let us consider the glide reflection $G_{y}=\left\{\sigma_{y} \mid \boldsymbol{\tau}_{x}\right\}$. A Bloch state $c_{\boldsymbol{k} m s}^{\dagger}$ is transformed as $\left(k_{x}, k_{y}, k_{z}\right) \rightarrow$ $\left(k_{x},-k_{y}, k_{z}\right),\left(s_{x}, s_{y}, s_{z}\right) \rightarrow\left(-s_{x}, s_{y},-s_{z}\right)$, and sublattice indices $(a, b) \rightarrow(b, a)$, and then the representation matrices are given by $D_{s^{\prime} s}^{(1 / 2)}\left(\sigma_{y}\right)=i \sigma_{y}^{\mathrm{T}}=\left(\begin{array}{cc}0 & -1 \\ 1 & 0\end{array}\right)$ and $D_{m^{\prime} m}^{(\mathrm{perm})}\left(\left\{\sigma_{y} \mid \boldsymbol{\tau}_{x}\right\} ; \boldsymbol{k}\right)=\left(\begin{array}{cc}0 & e^{-i\left(-k_{x} / 2-k_{y}\right)} \\ e^{-i\left(k_{x} / 2-k_{y}\right)} & 0\end{array}\right)$. Therefore, $G_{y}$ gives a relative phase factor $e^{i k_{x}}$ between the two sublattices. Intersublattice hybridization is forbidden at the zone face $k_{x}= \pm \pi$ owing to this phase factor. To prove it, we need to consider combined inversion-glide symmetry, which commutes with the Hamiltonian $\left[H, G_{y} I\right]=0$. This
TABLE I. List of characters for the IRs of the $D_{4 h}$ point group. The last column shows basis functions.

\begin{tabular}{lcrrrrrrrrrr}
\hline \hline & $E$ & $2 C_{4}$ & $C_{2}$ & $2 C_{2}^{\prime}$ & $2 C_{2}^{\prime \prime}$ & $I$ & $2 S_{4}$ & $\sigma_{h}$ & $2 \sigma_{v}$ & $2 \sigma_{d}$ & Basis functions \\
\hline$A_{1 g}$ & 1 & 1 & 1 & 1 & 1 & 1 & 1 & 1 & 1 & 1 & $k_{z}^{2}$ \\
$A_{2 g}$ & 1 & 1 & 1 & -1 & -1 & 1 & 1 & 1 & -1 & -1 & $k_{x} k_{y}\left(k_{x}^{2}-k_{y}^{2}\right)$ \\
$B_{1 g}$ & 1 & -1 & 1 & 1 & -1 & 1 & -1 & 1 & 1 & -1 & $k_{x}^{2}-k_{y}^{2}$ \\
$B_{2 g}$ & 1 & -1 & 1 & -1 & 1 & 1 & -1 & 1 & -1 & 1 & $k_{x} k_{y}$ \\
$E_{g}$ & 2 & 0 & -2 & 0 & 0 & 2 & 0 & -2 & 0 & 0 & $k_{z} k_{x}, k_{y} k_{z}$ \\
$A_{1 u}$ & 1 & 1 & 1 & 1 & 1 & -1 & -1 & -1 & -1 & -1 & $k_{x} \hat{\boldsymbol{x}}+k_{y} \hat{\boldsymbol{y}}$ \\
$A_{2 u}$ & 1 & 1 & 1 & -1 & -1 & -1 & -1 & -1 & 1 & 1 & $k_{y} \hat{\boldsymbol{x}}-k_{x} \hat{\boldsymbol{y}}$ \\
$B_{1 u}$ & 1 & -1 & 1 & 1 & -1 & -1 & 1 & -1 & -1 & 1 & $k_{x} \hat{\boldsymbol{x}}-k_{y} \hat{\boldsymbol{y}}$ \\
$B_{2 u}$ & 1 & -1 & 1 & -1 & 1 & -1 & 1 & -1 & 1 & -1 & $k_{y} \hat{\boldsymbol{x}}+k_{x} \hat{\boldsymbol{y}}$ \\
$E_{u}$ & 2 & 0 & -2 & 0 & 0 & -2 & 0 & 2 & 0 & 0 & $k_{x} \hat{\boldsymbol{z}}, k_{y} \hat{\boldsymbol{z}}$ \\
\hline \hline
\end{tabular}

imposes a constraint for intersublattice hybridization

$$
\begin{aligned}
\varepsilon_{1}(\boldsymbol{k}) & =\langle\boldsymbol{k} a s|H| \boldsymbol{k} b s\rangle \\
& =\left\langle\boldsymbol{k} a s\left|\left(G_{y} I\right)^{-1} G_{y} I H\left(G_{y} I\right)^{-1} G_{y} I\right| \boldsymbol{k} b s\right\rangle \\
& =\left(\left\langle\left(-k_{x}, k_{y}\right) a s\right| e^{i\left(k_{x} / 2-k_{y}\right)}\right) H \\
& \times\left(e^{-i\left(-k_{x} / 2-k_{y}\right)}\left|\left(-k_{x}, k_{y}\right) b s\right\rangle\right) \\
& =e^{i k_{x}}\left\langle\left(-k_{x}, k_{y}\right) a s|H|\left(-k_{x}, k_{y}\right) b s\right\rangle \\
& =e^{i k_{x}} \varepsilon_{1}\left(-k_{x}, k_{y}\right) .
\end{aligned}
$$

Then, the phase factor is $e^{i k_{x}}=-1$ on the zone face $k_{x}=$ $\pm \pi$, and therefore the intersublattice hybridization must be zero.

The space group $G$ in Eq. (8) can be reduced to a subgroup $G_{\text {intra }}$ by restricting to sublattice-conserving operations (see Table II)

$$
\begin{aligned}
G_{\text {intra }} & =\{E \mid \mathbf{0}\} T+\left\{2_{z} \mid \boldsymbol{\tau}_{x}+\boldsymbol{\tau}_{y}\right\} T \\
& +\left\{2_{x} \mid \boldsymbol{\tau}_{y}\right\} T+\left\{2_{y} \mid \boldsymbol{\tau}_{x}\right\} T \\
& +\left\{\sigma_{110} \mid \boldsymbol{\tau}_{x}+\boldsymbol{\tau}_{y}\right\} T+\left\{\sigma_{1-10} \mid \mathbf{0}\right\} T \\
& +\left\{I 4_{z}^{+} \mid \boldsymbol{\tau}_{\boldsymbol{x}}\right\} T+\left\{I 4_{z}^{-} \mid \boldsymbol{\tau}_{\boldsymbol{y}}\right\} T .
\end{aligned}
$$

From $G_{\text {intra }}$, we notice that the local site symmetry is $D_{2 d}$. Thus, parity mixing in intrasublattice pair amplitudes is allowed by the symmetry reduction $D_{4 h} \rightarrow D_{2 d}$, which is determined by the compatibility relation shown in Table III. Note that this simple rule is only applicable to the intrasublattice components.

For instance, the $B_{2 g}$ IR mixes with the $A_{2 u}$ IR because they are reduced to the same $B_{2}$ IR in $D_{2 d}$. The admixture is, however, different from that in the globally NCS superconductors: (1) One of the admixed components has a staggered form between sublattices for an intrasublattice pairing, and (2) parity mixing in intersublattice components is forbidden, to preserve the global inversion symmetry. These properties of the pair amplitude in locally NCS superconductors are derived from Eq. (6); the inversion symmetry represented by $D_{s_{1} s_{2} s s^{\prime}}^{(1 / 2)}(I)=\hat{1}_{4 \times 4}$ and $D_{m_{1} m_{2} m m^{\prime}}^{(\mathrm{perm})}(\{I \mid \mathbf{0}\})=\left(\begin{array}{cc}0 & \tau_{x} \\ \tau_{x} & 0\end{array}\right)$ 
TABLE II. List of permutation for each symmetry operation. The sublattice-conserving operations are labeled by a check mark $(\checkmark)$.

\begin{tabular}{|c|c|c|c|}
\hline & $a$ & $b$ & Sublattice conservation \\
\hline$\{E \mid \mathbf{0}\}$ & $a$ & $b$ & $\checkmark$ \\
\hline$\{I \mid \mathbf{0}\}$ & $b$ & $a$ & $x$ \\
\hline$\left\{2_{z} \mid \boldsymbol{\tau}_{x}+\boldsymbol{\tau}_{y}\right\}$ & $a$ & $b$ & $\checkmark$ \\
\hline$\left\{2_{x} \mid \boldsymbol{\tau}_{y}\right\}$ & $a$ & $b$ & $\checkmark$ \\
\hline$\left\{2_{y} \mid \boldsymbol{\tau}_{x}\right\}$ & $a$ & $b$ & $\checkmark$ \\
\hline$\left\{4_{z}^{+} \mid \boldsymbol{\tau}_{x}\right\}$ & $b$ & $a$ & $x$ \\
\hline$\left\{4_{z}^{-} \mid \boldsymbol{\tau}_{y}\right\}$ & $b$ & $a$ & $x$ \\
\hline$\left\{\sigma_{x} \mid \boldsymbol{\tau}_{y}\right\}$ & $b$ & $a$ & $x$ \\
\hline$\left\{\sigma_{y} \mid \boldsymbol{\tau}_{x}\right\}$ & $b$ & $a$ & $x$ \\
\hline$\left\{\sigma_{z} \mid \boldsymbol{\tau}_{x}+\boldsymbol{\tau}_{y}\right\}$ & $b$ & $a$ & $x$ \\
\hline$\left\{\sigma_{110} \mid \boldsymbol{\tau}_{x}+\boldsymbol{\tau}_{y}\right\}$ & $a$ & $b$ & $\checkmark$ \\
\hline$\left\{\sigma_{1-10} \mid \mathbf{0}\right\}$ & $a$ & $b$ & $\checkmark$ \\
\hline$\left\{2_{110} \mid \mathbf{0}\right\}$ & $b$ & $a$ & $x$ \\
\hline$\left\{2_{1-10} \mid \boldsymbol{\tau}_{x}+\boldsymbol{\tau}_{y}\right\}$ & $b$ & $a$ & $x$ \\
\hline$\left\{I 4_{z}^{+} \mid \boldsymbol{\tau}_{\boldsymbol{x}}\right\}$ & $a$ & $b$ & $\checkmark$ \\
\hline$\left\{I 4_{z}^{-} \mid \tau_{y}\right\}$ & $a$ & $b$ & $\checkmark$ \\
\hline
\end{tabular}

TABLE III. Reduction of IRs $D_{4 h} \rightarrow D_{2 d}$. The two fold rotational symmetry axes of $D_{2 d}$ are the $x / y$ axes of $D_{4 h}$.

\begin{tabular}{lcccccccccc}
\hline$D_{4 h}$ & $A_{1 g}$ & $A_{2 g}$ & $B_{1 g}$ & $B_{2 g}$ & $E_{g}$ & $A_{1 u}$ & $A_{2 u}$ & $B_{1 u}$ & $B_{2 u}$ & $E_{u}$ \\
\hline$D_{4 h} \downarrow D_{2 d}$ & $A_{1}$ & $A_{2}$ & $B_{1}$ & $B_{2}$ & $E$ & $B_{1}$ & $B_{2}$ & $A_{1}$ & $A_{2}$ & $E$ \\
\hline \hline
\end{tabular}

imposes a constraint for intra- and inter-sublattice pair amplitudes

$$
\begin{aligned}
& I F_{a s a s^{\prime}}^{\Gamma_{g(u)}}(\boldsymbol{k}) I^{-1}=+(-) F_{b s b s^{\prime}}^{\Gamma_{g(u)}}(-\boldsymbol{k})=-(+) F_{b s^{\prime} b s}^{\Gamma_{g(u)}}(\boldsymbol{k}), \\
& I F_{a s b s^{\prime}}^{\Gamma_{g(u)}}(\boldsymbol{k}) I^{-1}=+(-) F_{b s a s^{\prime}}^{\Gamma_{g(u)}}(-\boldsymbol{k})=-(+) F_{a s^{\prime} b s}^{\Gamma_{g(u)}}(\boldsymbol{k}),
\end{aligned}
$$

for even-parity (odd-parity) superconductivity.

For example, let us consider the even-parity superconductivity, for which a part of Eqs. (11) and (12) are explicitly described as

$$
\begin{gathered}
I F_{a \uparrow a \uparrow}^{\Gamma_{g}}(\boldsymbol{k}) I^{-1}=F_{b \uparrow b \uparrow}^{\Gamma_{g}}(-\boldsymbol{k})=-F_{b \uparrow b \uparrow}^{\Gamma_{g}}(\boldsymbol{k}), \\
I F_{a \uparrow a \downarrow}^{\Gamma_{g}}(\boldsymbol{k}) I^{-1}=F_{b \uparrow b \downarrow}^{\Gamma_{g}}(-\boldsymbol{k})=-F_{b \downarrow b \uparrow}^{\Gamma_{g}}(\boldsymbol{k}), \\
I F_{a \uparrow b \uparrow}^{\Gamma_{g}}(\boldsymbol{k}) I^{-1}=F_{b \uparrow a \uparrow}^{\Gamma_{g}}(-\boldsymbol{k})=-F_{a \uparrow b \uparrow}^{\Gamma_{g}}(\boldsymbol{k}), \\
I F_{a \uparrow b \downarrow}^{\Gamma_{g}}(\boldsymbol{k}) I^{-1}=F_{b \uparrow a \downarrow}^{\Gamma_{g}}(-\boldsymbol{k})=-F_{a \downarrow b \uparrow}^{\Gamma_{g}}(\boldsymbol{k}) .
\end{gathered}
$$

Equation (13) shows a symmetry property of the $S_{z}=1$ intrasublattice Cooper pair, namely, parity-mixed spin-triplet pairing. This equation means that the exchange of sublattice indices $(a \rightarrow b)$ gives a sign change of pair amplitude $F_{a \uparrow a \uparrow}^{\Gamma_{g}}(\boldsymbol{k})=-F_{b \uparrow b \uparrow}^{\Gamma_{g}}(\boldsymbol{k})$. Thus, the parity-mixed intrasublattice component has a staggered form. Equation (15) indicates a relation of the parity-mixed intersublattice pairing
$F_{a \uparrow b \uparrow}^{\Gamma_{g}}(\boldsymbol{k})=-F_{a \uparrow b \uparrow}^{\Gamma_{g}}(\boldsymbol{k})=0$. We immediately find that the parity-mixed intersublattice component must be zero.

Recent progress on the group-theoretical analysis of superconductivity has shown unusual nodal/gapped structures ensured by nonsymmorphic symmetry [83-90]. Similarly, we show peculiar structures of the pair amplitude. The spacegroup symmetry of our model (8) is nonsymmorphic since it contains the glide symmetry.

Let us consider the glide symmetry $G_{x}=\left\{\sigma_{x} \mid \tau_{y}\right\}$ represented by

$$
\begin{aligned}
D_{s_{1} s_{2} s s^{\prime}}^{(1 / 2)}\left(\sigma_{x}\right) & =\left(\begin{array}{rr} 
& -\sigma_{x} \\
-\sigma_{x} &
\end{array}\right), \\
D_{m_{1} m_{2} m m^{\prime}}^{(\text {perm })}\left(\left\{\sigma_{x} \mid \tau_{y}\right\}\right) & =\left(\begin{array}{cc} 
& 1 \\
e^{-i k_{y}} &
\end{array}\right) .
\end{aligned}
$$

We especially focus on intersublattice Cooper pairs on the zone face $k_{y}= \pm \pi$ at $k_{z}=0$. On this high-symmetry line, $G_{x}$ imposes a constraint,

$$
G_{x} F_{a \uparrow b \downarrow}^{\Gamma_{i}}\left(k_{x}, \pi, 0\right) G_{x}^{-1}=e^{-i \pi} D^{\phi_{k}}\left(\sigma_{x} ; \Gamma_{i}\right) F_{a \uparrow b \downarrow}^{\Gamma_{i}}\left(k_{x},-\pi, 0\right) .
$$

Therefore, it is indicated that intersublattice gap functions must be zero for glide-even superconducting states. For instance, we find that the intersublattice pair amplitudes in the $A_{1 g}$ IR ( $s$-wave state) and $B_{1 g}$ IR $\left(d_{x^{2}-y^{2}}\right.$-wave state) have nodal lines at $k_{z}=0$ and $k_{x, y}= \pm \pi$. On the other hand, the pair amplitude is finite for glide-odd IRs such as $B_{2 g}$ IR $\left(d_{x y^{-}}\right.$ wave state). These features are opposite to those expected from a group-theoretical analysis of symmorphic superconductors [80].

In the following sections, we study superconductivity in a model preserving the space group symmetry (8). The gap functions obtained from numerical calculations satisfy the symmetry constraints, which have been revealed in this section.

\section{MODEL AND METHOD}

In this section, we introduce a two-dimensional twosublattice Hubbard model which was adopted for $\mathrm{Sr}_{2} \mathrm{IrO}_{4}$ [39]. We consider a two-dimensional $\mathrm{IrO}_{2}$ plane of quasitwo-dimensional $\mathrm{Sr}_{2} \mathrm{IrO}_{4}$. The crystal structure has been illustrated in Fig. 1(a). We do not restrict our discussions to $\mathrm{Sr}_{2} \mathrm{IrO}_{4}$ and later propose some other candidate materials. However, it is significant to study a well-studied model for $\mathrm{Sr}_{2} \mathrm{IrO}_{4}$ as a typical example and to illustrate the effects of spin-orbit coupling and multipole fluctuations.

The total Hamiltonian is written as $H=H_{0}+H_{\text {int }}+$ 
$H_{\mathrm{ASOC}}$. The Hamiltonian of kinetic energy terms is

$$
\begin{aligned}
H_{0} & =\sum_{\boldsymbol{k} s} \sum_{m \neq m^{\prime}}\left(\varepsilon_{1}(\boldsymbol{k}) c_{\boldsymbol{k} m s}^{\dagger} c_{\boldsymbol{k} m^{\prime} s}+\text { H.c. }\right) \\
& +\sum_{\boldsymbol{k} m s} \varepsilon_{2}(\boldsymbol{k}) c_{\boldsymbol{k} m s}^{\dagger} c_{\boldsymbol{k} m s},
\end{aligned}
$$

where $c_{\boldsymbol{k} m s}^{(\dagger)}$ is the annihilation (creation) operator of an Ir$5 d$ electron with pseudospin $s$ on sublattice $m=(a, b)$. The pseudospin corresponds to the $j_{\text {eff }}=1 / 2$ doublet states formed by a strong spin-orbit coupling [50]. The singleelectron kinetic energy is described by taking into account the nearest- and next-nearest-neighbor hoppings,

$$
\begin{aligned}
& \varepsilon_{1}(\boldsymbol{k})=-t_{1}\left(1+e^{i k_{x}}\right)\left(1+e^{-i k_{y}}\right), \\
& \varepsilon_{2}(\boldsymbol{k})=-2 t_{2}\left(\cos k_{x}+\cos k_{y}\right) .
\end{aligned}
$$

The on-site Coulomb interaction on an Ir site is given by

$$
H_{\mathrm{int}}=U \sum_{i m} n_{i m \uparrow} n_{i m \downarrow} .
$$

The ASOC term is written as

$$
H_{\mathrm{ASOC}}=\alpha \sum_{\substack{\boldsymbol{k} s s^{\prime} \\ m m^{\prime}}} \boldsymbol{g}(\boldsymbol{k}) \cdot \boldsymbol{\sigma}_{s s^{\prime}} c_{\boldsymbol{k} m s}^{\dagger} c_{\boldsymbol{k} m^{\prime} s^{\prime}} \zeta_{m m^{\prime}}^{z},
$$

where $\zeta^{\mu}$ is a Pauli matrix for sublattice degrees of freedom. We consider the staggered ASOC arising from the spindependent intrasublattice hopping,

$$
\boldsymbol{g}(\boldsymbol{k})=\sin k_{x} \cos k_{y} \hat{x}-\sin k_{y} \cos k_{x} \hat{y} .
$$

Superconductivity in this model is investigated by solving the linearized Eliashberg equation,

$$
\begin{aligned}
\lambda \Delta_{\xi \xi^{\prime}}(k) & =-\frac{T}{N} \sum_{k^{\prime}} \sum_{\xi_{1} \xi_{2} \xi_{3} \xi_{4}} V_{\xi \xi_{1}, \xi_{2} \xi^{\prime}}\left(k-k^{\prime}\right) \\
& \times G_{\xi_{3} \xi_{1}}\left(-k^{\prime}\right) \Delta_{\xi_{3} \xi_{4}}\left(k^{\prime}\right) G_{\xi_{4} \xi_{2}}\left(k^{\prime}\right),
\end{aligned}
$$

where $\hat{G}(k)=\left[\left(i \varepsilon_{m}-\mu\right) \hat{1}-\hat{H}(\boldsymbol{k})\right]^{-1}$ and $i \varepsilon_{m}=i(2 m+$ 1) $\pi T$ is the fermionic Matsubara frequency. Here, we use abbreviated notations $k=\left(\boldsymbol{k}, i \varepsilon_{m}\right)$ and $\xi=(m, s)$. In the RPA, effective pairing interaction is described by the generalized susceptibility in the $8 \times 8$ matrix,

$$
\hat{V}(q)=-\hat{\Gamma}^{0} \hat{\chi}(q) \hat{\Gamma}^{0}-\hat{\Gamma}^{0} .
$$

In the two-sublattice single-orbital model, the bare irreducible vertex is obtained as

$$
\begin{aligned}
\Gamma_{m s_{1} m s_{2}, m^{\prime} s_{3} m^{\prime} s_{4}}^{0} & =\frac{1}{2} \Gamma_{m m^{\prime}}^{s} \boldsymbol{\sigma}_{s_{1} s_{2}} \cdot \boldsymbol{\sigma}_{s_{4} s_{3}} \\
& -\frac{1}{2} \Gamma_{m m^{\prime}}^{c} \delta_{s_{1} s_{2}} \delta_{s_{4} s_{3}},
\end{aligned}
$$

and $\hat{\Gamma}_{m m^{\prime}}^{s(c)}=U \delta_{m m^{\prime}}$. The RPA susceptibility is given by

$$
\hat{\chi}(q)=\hat{\chi}^{0}(q)\left[\hat{1}-\hat{\Gamma}^{0} \hat{\chi}^{0}(q)\right]^{-1},
$$

where the irreducible susceptibility is defined as $\hat{\chi}^{0}(q)=$ $-(T / N) \sum_{k} \hat{G}(k+q) \hat{G}(k)$. Now we introduce the magnetic susceptibilities

$$
\chi_{m m^{\prime}}^{\mu \nu}(q)=\sum_{s_{1} s_{2} s_{3} s_{4}} \sigma_{s_{1} s_{2}}^{\mu} \chi_{m s_{1} m s_{2}, m^{\prime} s_{3} m^{\prime} s_{4}}(q) \sigma_{s_{4} s_{3}}^{\nu},
$$

where $\mu, \nu=x, y, z$. The magnetic fluctuation parallel (perpendicular) to the $c$ axis $\chi_{m m^{\prime}}^{\|}(q)\left[\chi_{m m^{\prime}}^{\perp}(q)\right]$ is characterized by $\chi_{m m^{\prime}}^{\|}(q) \equiv \chi_{m m^{\prime}}^{z z}(q)\left[\chi_{m m^{\prime}}^{\perp}(q) \equiv\right.$ $\left.\left(\chi_{m m^{\prime}}^{x x}(q)+\chi_{m m^{\prime}}^{y y}(q)\right) / 2\right]$.

We define the band filling $n$ as the number of electrons per unit cell (e.g., $n=4$ for full filling). The doping level $x$ is related to the band filling as $n=2+2 x$. A variational Monte Carlo study [60] for $\mathrm{Sr}_{2} \mathrm{IrO}_{4}$ shows that the $d$-wave superconducting state is stable near the doping level $x=0.2$. Thus, we study $x=0.2$ unless mentioned otherwise. We also discuss the result in the undoped case $x=0$. We set $\left(t_{1}, t_{2}\right)=(1.0,0.26), T=0.02,64 \times 64 \boldsymbol{k}$-point meshes, and 1024 Matsubara frequencies in the numerical calculations.

\section{MAGNETIC FLUCTUATION}

First, we study the magnetic fluctuation. When ASOC is absent, the magnetic anisotropy does not exist, and therefore we have $\chi^{\|}=\chi^{\perp}$. Then, the two sublattices are equivalent, and the model is equivalent to the ordinary single-sublattice Hubbard model which has been studied for a long time [91]. Near half-filling, the AFM fluctuation with a wave vector around $\boldsymbol{q}=(\pi, \pi)$ is enhanced. On the other hand, when the ASOC is turned on, the two sublattices are nonequivalent, and the Brillouin zone is folded. In the folded Brillouin zone, the wave vector is $\boldsymbol{q}=\mathbf{0}$, indicating a ferroic multipole fluctuation. In our model, the nesting of the Fermi surface gives rise to the magnetic correlation parallel to the $c$ axis at $\boldsymbol{q}=\mathbf{0}$ for $\alpha<0.2$ as shown in Fig. 2(a). The anisotropy of the Ising-like magnetic fluctuation is compatible with the magnetic structure in $\mathrm{BaMn}_{2} \mathrm{As}_{2}[28,48]$, which possesses a weak spin-orbit coupling. For $\alpha>0.2$, the Ising-like magnetic fluctuation is significantly suppressed by ASOC, and the incommensurate magnetic correlation perpendicular to the $c$ axis becomes predominant. This in-plane magnetic anisotropy is consistent with the $5 d$ transition metal oxide $\mathrm{Sr}_{2} \mathrm{IrO}_{4}$ having strong spin-orbit coupling [39, 40, 49-62]. Thus, the model captures qualitative properties of these materials although a significantly simplified model is adopted. The suppression of magnetic fluctuation by the ASOC is a generic feature [16], and it has been confirmed by a NMR experiment in $\mathrm{CeCoIn}_{5}$ superlattices [92]. A qualitatively same conclusion is obtained from noninteracting magnetic susceptibility (see Fig. 3).

The ASOC dependence of the magnetic anisotropy may be attributed to the Fermi surface around the $M$ point. Figures 2(c) and 2(d) show the band structure and Fermi surfaces with a spin texture for $\alpha=0.1$ and 0.3 , respectively. We find a Lifshitz transition at $\alpha \sim 0.2$, involving the change of the spin texture on the Fermi surface. Since the intersublattice hopping disappears on the $X-M$ line as ensured by nonsymmorphic crystal symmetry, we can define the spin texture on 
(a)

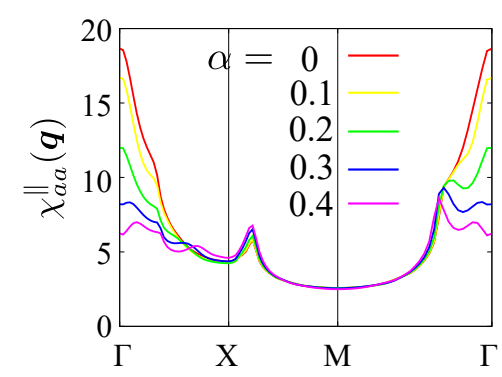

(b)

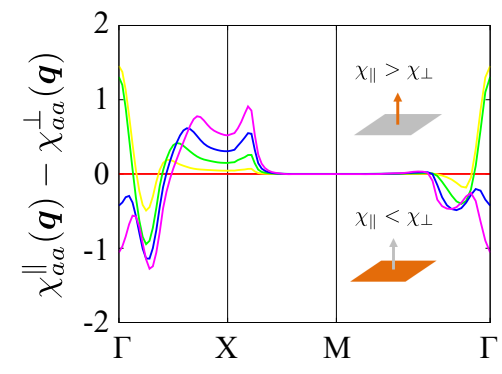

(c)
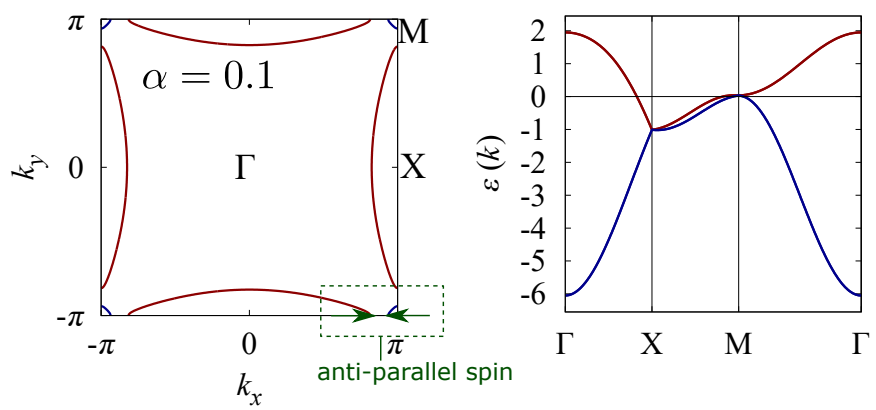

(d)
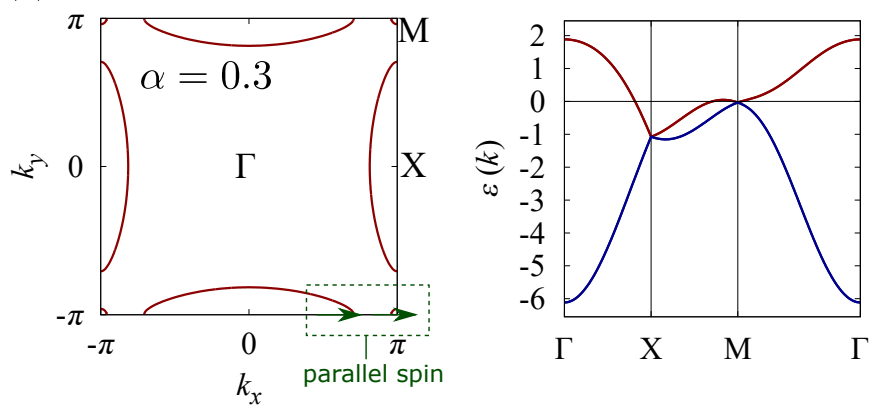

FIG. 2. (a), (b) Momentum dependence of the magnetic susceptibility. A matrix element of the static susceptibility $\chi_{a a(=b b)}^{\|, \perp}\left(\boldsymbol{q}, i \omega_{n}=0\right)$ on the symmetry axes is shown. (a) shows $\chi^{\|}$, while (b) shows the anisotropy $\chi^{\|}-\chi^{\perp}$ for $U=1.8, x=0.2$, and $T=0.02$. (c)-(d) Momentum dependence of the band dispersion (right panels) and Fermi surfaces (left panels) for $\alpha=0.1$ and 0.3 . The arrows show the spin texture on a sublattice.

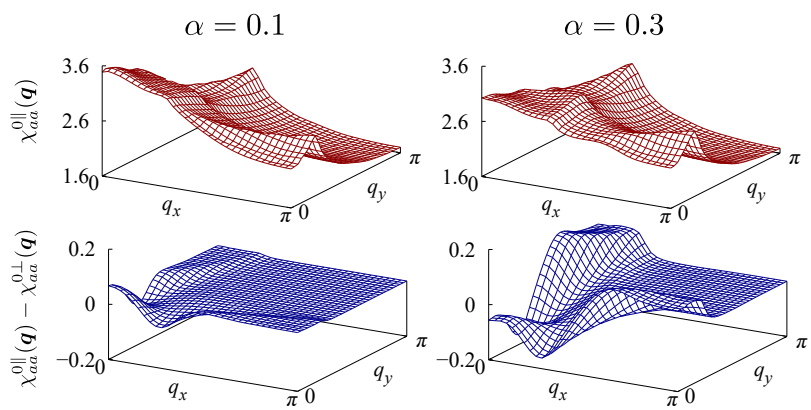

FIG. 3. Momentum dependence of the magnetic susceptibility for $U=0$ (irreducible bubble susceptibility).

each sublattice. As we show in the left panels of Figs. 2(c) and 2(d), the spin texture is antiparallel (parallel) between the two Fermi surfaces for $\alpha=0.1(\alpha=0.3)$. The change in magnetic anisotropy around $\boldsymbol{q}=\mathbf{0}$ coincides with the Lifshitz transition. Note that for the doping level $x=0$, the ASOCinduced Lifshitz transition does not occur up to $\alpha=1$, and then, the anisotropy is always $\chi^{\|}>\chi^{\perp}$. Thus, it is implied that the change in the magnetic anisotropy is related to the Lifshitz transition.

Next, we classify the magnetic fluctuations into augmented cluster multipole fluctuations on the basis of the group the- ory [28]. When the AFM transition of $\boldsymbol{m} \| c$ occurs, the crystal symmetry of $D_{4 h}$ is reduced to the subgroup $D_{2 d}^{\prime}$, in which the twofold rotational symmetry axes are rotated $45^{\circ}$ from $D_{2 d}$. The IRs of $D_{4 h}$ are also reduced to representations of $D_{2 d}^{\prime}$. Since only the $B_{2 u}$ IR contains the fully symmetric $A_{1}$ IR of $D_{2 d}^{\prime}$, the magnetic order belongs to the $B_{2 u}$ IR of $D_{4 h}$. A basis function of the $B_{2 u}$ IR is a linear combination of magnetic quadrupole and hexadecapole moments. On the other hand, the AFM structure of $\boldsymbol{m} \perp c$ reduces $D_{4 h}$ to $C_{2 v}$. The $E_{u}$ IR is the candidate of the order parameter. A basis function of the $E_{u}$ IR contains magnetic quadrupole and toroidal moments. Both $B_{2 u}$ and $E_{u}$ IRs represent odd-parity orders, which spontaneously break global inversion symmetry. Thus, odd-parity multipole fluctuations are enhanced in our model.

To clarify the multipole fluctuations, we calculate the odd-parity multipole susceptibility defined as $\chi_{B_{2 u}\left(E_{u}\right)}=$ $\sum_{m m^{\prime}} \chi_{m m^{\prime}}^{\|(\perp)}\left(\zeta_{m m^{\prime}}^{0}-\zeta_{m m^{\prime}}^{x}\right)$, where $\zeta^{\mu}$ is a Pauli matrix for sublattice degree of freedom [93]. Here, the magnetic representation is systematically derived from $\Gamma_{\text {tot }}=\Gamma_{\text {mag }} \otimes$ $\Gamma_{\text {sub }}=A_{2 g}\left(E_{g}\right) \otimes B_{1 u}=B_{2 u}\left(E_{u}\right)$, where $\Gamma_{\text {sub }}$ is an induced representation of $\left(A_{1}\right.$ of $\left.D_{2 d}\right) \uparrow D_{4 h}$ except for the fully symmetric representation of $D_{4 h}$. When $\chi_{B_{2 u}\left(E_{u}\right)}$ diverges at $\boldsymbol{q}=\mathbf{0}$, an odd-parity magnetic multipole order accompanied by inversion symmetry breaking occurs. Indeed, the sublattice off-diagonal components $\chi_{a b}\left(=\chi_{b a}\right)$ are nega- 


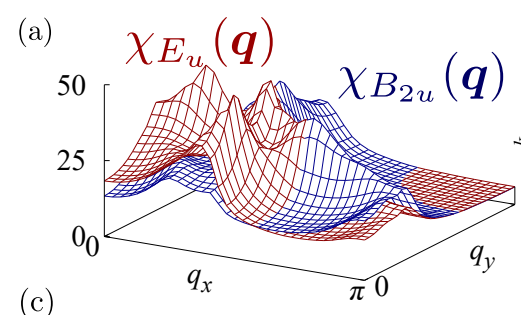

(c)

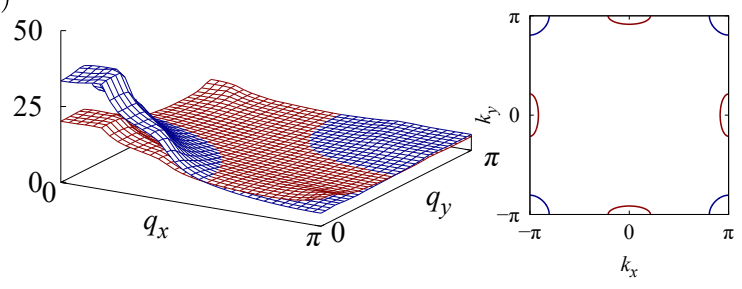

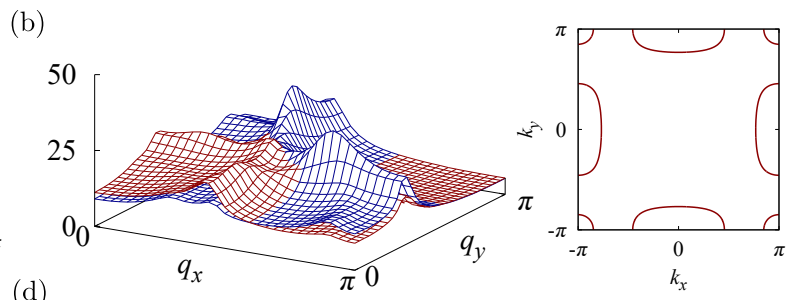

(d)

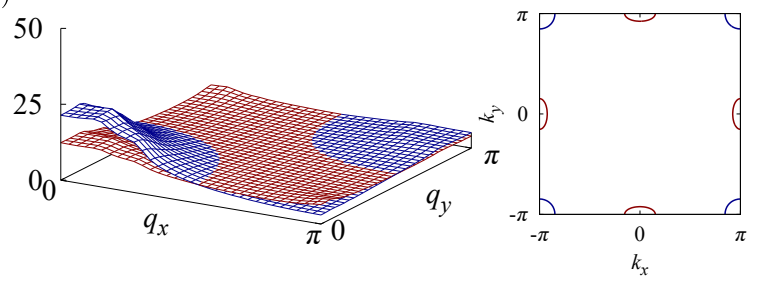

FIG. 4. Momentum dependence of the multipole susceptibilities $\chi_{E u}(\boldsymbol{q})$ and $\chi_{B 2 u}(\boldsymbol{q})$ for $U=2.2$. (a) $\alpha=0.7$ and $x=0.2$, (b) $\alpha=1$ and $x=0.2$, (c) $\alpha=0.7$ and $x=0$, and (d) $\alpha=1$ and $x=0$. The right panels show the Fermi surfaces.

tively enhanced, and therefore $\chi_{B_{2 u}\left(E_{u}\right)}$ diverges by increasing $U$, which is consistent with the analysis of the eigenvector of $\hat{\chi}^{0}(\boldsymbol{q}) \hat{\Gamma}^{0}$. Figures 4(a) and 4(b) show $\boldsymbol{q}$ dependence of $\chi_{B_{2 u}\left(E_{u}\right)}$ for $x=0.2$. Here, we adopt a large ASOC since we discuss unconventional superconductivity in this region later. As we have shown in Fig. 2, for a large ASOC the wave vector $\boldsymbol{q}$ of the magnetic order is finite at $x=0.2$. We call such incommensurate order the quadrupole density wave in a broad sense. On the other hand, incommensurate susceptibility is not enhanced in the undoped case $(x=0)$ [Figs. 4(c) and 4(d)]. Then, the ferroic multipole fluctuation of $\chi_{B_{2 u}}$ is predominant because of the absence of a specific nesting in Fermi surfaces. These odd-parity fluctuations affect superconductivity, as we demonstrate in the next section.

\section{SUPERCONDUCTIVITY}

Here, we examine superconductivity. Although this work is based on a model motivated by $\mathrm{BaMn}_{2} \mathrm{As}_{2}$ and $\mathrm{Sr}_{2} \mathrm{IrO}_{4}$, the following results are qualitatively valid in a broad range of odd-parity magnetic multipole materials which have been recently identified [75].

Before showing the numerical results of the Eliashberg equation, we discuss the effects of ASOC on superconductivity in locally NCS systems. The ASOC has two effects: (1) modulation of the one-particle Green's function, and (2) that of the pairing interaction. Considering effect (1), we may recognize that the stable superconducting state depends on whether the leading pairing channel is the intrasublattice pairing or intersublattice pairing (see Fig. 5 for an illustration) [3]. This gives a selection rule summarized in Table IV. The spin-singlet pairing state or spin-triplet pairing state with $\boldsymbol{d}(\boldsymbol{k}) \| \boldsymbol{g}(\boldsymbol{k})$ are stable for intrasublattice pairing, while only the spin-triplet pairing state with $\boldsymbol{d}(\boldsymbol{k}) \perp \boldsymbol{g}(\boldsymbol{k})$ is stable for intersublattice pairing. The other superconducting states are destabilized by sublattice-dependent ASOC. Although the se- lection rule for intrasublattice pairing is equivalent to that of globally NCS superconductors, the selection rule for intersublattice pairing is peculiar to locally NCS superconductors. We may understand the selection rule with the help of the band structure in Fig. 5. The effect of ASOC on the band structure is taken into account through the one-particle Green's function. On the other hand, effect (2) occurs through the modification of magnetic fluctuation, which has been investigated in Sec. V. Later, we show that the modified magnetic fluctuation stabilizes odd-parity spin-triplet superconductivity.

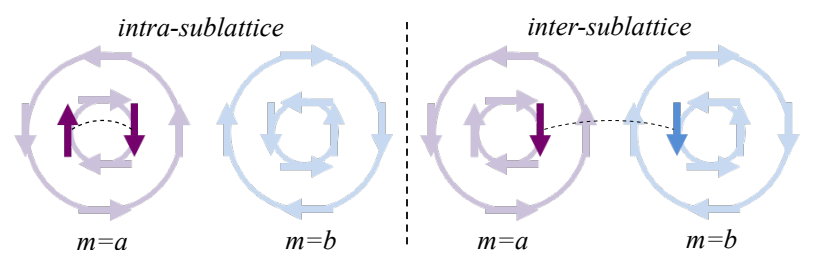

FIG. 5. Schematic figure of Fermi surfaces in a bilayer Rashba system. In the absence of intersublattice hybridization, the spin texture on each sublattice can be defined. In locally NCS systems, sublattices $a$ and $b$ have opposite spin textures. Left panel: For intrasublattice pairing, Cooper pairs with total spin $S_{z}=0$, namely, the spin-singlet state and spin-triplet state with $\boldsymbol{d}(\boldsymbol{k}) \| \boldsymbol{g}(\boldsymbol{k})$, are preferred, while other states are suppressed. Right panel: For intersublattice pairing, Cooper pairing with $S_{z}=1$ corresponding to the spin-triplet state with $\boldsymbol{d}(\boldsymbol{k}) \perp \boldsymbol{g}(\boldsymbol{k})$ is stable. The spin-singlet pairing and spin-triplet pairing state with $\boldsymbol{d}(\boldsymbol{k}) \| \boldsymbol{g}(\boldsymbol{k})$ are fragile by the ASOC.

Now we show the numerical results. Within the RPA theory, we obtained four stable superconducting states: $B_{2 g}$, $B_{1 u}, B_{1 g}$, and $A_{1 u}$ IRs. The leading pair amplitude and other admixed components of the superconducting states are summarized in Table V. As we have shown in Sec. III, parity mixing does not appear in intersublattice components. Thus, Table V illustrates the intrasublattice component, paritymixed intrasublattice component, and intersublattice com- 

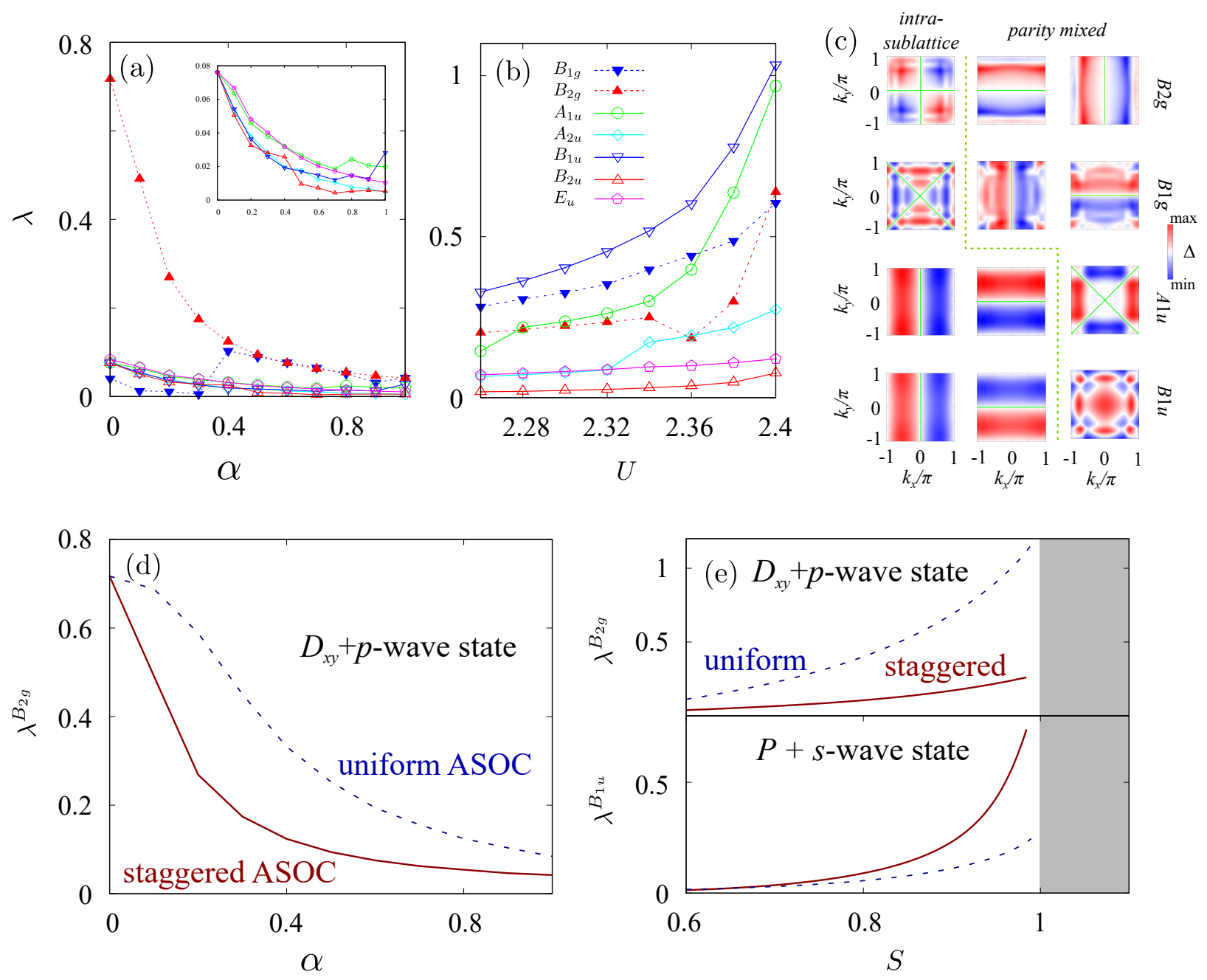

FIG. 6. (a) ASOC dependence of eigenvalues of the Eliashberg equation $\lambda$ for $U=1.6$ and $T=0.02$. The $B_{1 g}\left(d_{x^{2}-y^{2}}+p\right.$-wave state), $B_{2 g}\left(d_{x y}+p\right.$-wave state), $A_{1 u}\left(p+d_{x^{2}-y^{2}}\right.$-wave state), $A_{2 u}\left(p+d_{x y}\right.$-wave state $), B_{1 u}(p+s$-wave state $), B_{2 u}(p+g$-wave state $), E_{u}(p+$ $d_{z x, y z}$-wave state) IRs are depicted. (b) Coulomb interaction dependence of $\lambda$ for $\alpha=0.9$. (c) Intra-sublattice and parity-mixed components of gap functions for $U=1.4$ and $\alpha=0.1$. For the spin-triplet components, $d^{x 0}(\boldsymbol{k})$ and $d^{y 0}(\boldsymbol{k})$ are shown for the $A_{1 u}$ and $B_{1 u}$ IRs, while $d^{x z}(\boldsymbol{k})$ and $d^{y z}(\boldsymbol{k})$ are shown for the $B_{2 g}$ and $B_{1 g}$ IRs. (d) ASOC dependence of $\lambda^{B_{2 g}}$ for the $B_{2 g}$ IR at $U=1.6$. The solid line shows the result of our model introduced in Sec. VI for the locally NCS crystal. For comparison, we show the dashed line obtained for the model containing a uniform ASOC instead of the staggered ASOC. The latter corresponds to the globally NCS crystal. (e) Stoner factor $S=[\hat{\Gamma} \hat{\chi}(\boldsymbol{q})]_{\text {max }}$ dependence of $\lambda^{B_{2 g}}$ (upper panel) and $\lambda^{B_{1 u}}$ (lower panel) for $\alpha=0.9$. We again compare the locally NCS model (solid lines) with the globally NCS model (dashed lines).

TABLE IV. Selection rules of the superconductivity in locally and globally NCS crystals [3].

\begin{tabular}{lcc}
\hline \hline globally NCS crystal & \multicolumn{2}{c}{ locally NCS crystal } \\
\hline intrasublattice & intrasublattice & intersublattice \\
singlet, $\boldsymbol{d}(\boldsymbol{k}) \| \boldsymbol{g}(\boldsymbol{k})$ & singlet, $\boldsymbol{d}(\boldsymbol{k}) \| \boldsymbol{g}(\boldsymbol{k})$ & $\boldsymbol{d}(\boldsymbol{k}) \perp \boldsymbol{g}(\boldsymbol{k})$ \\
\hline \hline
\end{tabular}

ponent. For convenience we describe the order parameter of superconductivity in a standard manner, $\hat{\Delta}(\boldsymbol{k}, i \pi T)=$ $\sum_{\mu \nu} d^{\mu \nu}(\boldsymbol{k}) \bar{\sigma}_{s s^{\prime}}^{\mu} \zeta_{m m^{\prime}}^{\nu}$, where $\sigma^{\mu}$ and $\zeta^{\nu}$ are the Pauli matrix for spin and sublattice degrees of freedom, respectively. We introduced $\bar{\sigma}_{s s^{\prime}}^{\mu}=\left[\sigma^{\mu} i \sigma^{y}\right]_{s s^{\prime}}$ for $\mu=0, x, y, z$. This notation is used in Table V.

The $B_{2 g}$ state corresponds to the spin-singlet $d_{x^{2}-y^{2}}$-wave pairing state in the well-studied single-sublattice Hubbard model. Since the $x$ and $y$ axes in the two-sublattice model are rotated $45^{\circ}$, the predominant component of the order parameter is an intersublattice spin-singlet component $d^{0 x}(\boldsymbol{k})$ of $d_{x y}$-wave symmetry. Consistent with many theoretical works on the single-sublattice Hubbard model near half filling [91], the $B_{2 g}$ state is stable at $\alpha=0$. However, when the staggered ASOC is turned on, the eigenvalue of the Eliashberg equation $\lambda$ for the $B_{2 g}$ state is steeply suppressed [Fig. 6(a)]. This is mainly because intersublattice spin-singlet pairing is 
TABLE V. List of gap functions for the $B_{1 g}, B_{2 g}, A_{1 u}$, and $B_{1 u}$ superconducting states. Intrasublattice component $d^{\mu 0}(\boldsymbol{k})$, parity-mixed component $d^{\mu z}(\boldsymbol{k})$, and intersublattice component $d^{\mu x}(\boldsymbol{k})$ are listed. The last column shows the leading component.

\begin{tabular}{|c|c|c|c|c|c|c|}
\hline & Intrasublattice & & Parity mixed & & Intersublattice & Leading component \\
\hline$B_{1 g}$ & $\cos k_{x}-\cos k_{y}$ & $\zeta^{0} \bar{\sigma}^{0}$ & $\sin k_{x} \hat{\boldsymbol{x}}+\sin k_{y} \hat{\boldsymbol{y}}$ & $\zeta^{z} \bar{\sigma}^{x}, \zeta^{z} \bar{\sigma}^{y}$ & $\zeta^{x} \bar{\sigma}^{0}$ & $\zeta^{0} \bar{\sigma}^{0}$ \\
\hline$B_{2 g}$ & $\sin k_{x} \sin k_{y}$ & $\zeta^{0} \bar{\sigma}^{0}$ & $\sin k_{y} \hat{\boldsymbol{x}}-\sin k_{x} \hat{\boldsymbol{y}}$ & $\zeta^{z} \bar{\sigma}^{x}, \zeta^{z} \bar{\sigma}^{y}$ & $\zeta^{x} \bar{\sigma}^{0}$ & $\zeta^{x} \bar{\sigma}^{0}$ \\
\hline$A_{1 u}$ & $\sin k_{x} \hat{\boldsymbol{x}}+\sin k_{y} \hat{\boldsymbol{y}}$ & $\zeta^{0} \bar{\sigma}^{x}, \zeta^{0} \bar{\sigma}^{y}$ & $\cos k_{x}-\cos k_{y}$ & $\zeta^{z} \bar{\sigma}^{0}$ & $\zeta^{x} \bar{\sigma}^{x}, \zeta^{x} \bar{\sigma}^{y}$ & $\zeta^{0} \bar{\sigma}^{x}, \zeta^{0} \bar{\sigma}^{y}$ \\
\hline$B_{1 u}$ & $\sin k_{x} \hat{\boldsymbol{x}}-\sin k_{y} \hat{\boldsymbol{y}}$ & $\zeta^{0} \bar{\sigma}^{x}, \zeta^{0} \bar{\sigma}^{y}$ & $\cos k_{x}+\cos k_{y}$ & $\zeta^{z} \bar{\sigma}^{0}$ & $\zeta^{x} \bar{\sigma}^{x}, \zeta^{x} \bar{\sigma}^{y}$ & $\zeta^{0} \bar{\sigma}^{x}, \zeta^{0} \bar{\sigma}^{y}$ \\
\hline
\end{tabular}

ruled out by the selection rule of locally NCS superconductors, in striking contrast to the globally NCS superconductors (see Table IV).

To examine the effect of the staggered ASOC, we solved the Eliashberg equation for a similar model containing a uniform ASOC instead of the staggered ASOC. The eigenvalues $\lambda$ of the two models are compared in Fig. 6(d). Consistent with the selection rule, the local parity violation more significantly suppresses $d$-wave superconductivity than does the global parity violation. In both cases, superconductivity is suppressed by ASOC owing to the suppressed magnetic fluctuation. In addition, the staggered ASOC causes pair breaking through the modulation of the one-particle Green's function. The dominant pairing component $d^{0 x}(\boldsymbol{k})$, which is incompatible with the selection rule in Table IV, decreases in the same manner as $\lambda^{B_{2 g}}$ by increasing $\alpha$. Instead of that, an intrasublattice spin-singlet component compatible with the selection rule monotonically increases as $d^{00}(\boldsymbol{k}) \simeq$ $\left.d^{00}(\boldsymbol{k})\right|_{\alpha=0}+A \alpha \sin k_{x} \sin k_{y}$. Owing to the parity mixing by the ASOC, an admixed staggered spin-triplet component, $\left(d^{x z}(\boldsymbol{k}), d^{y z}(\boldsymbol{k})\right) \simeq B \alpha\left(\sin k_{y},-\sin k_{x}\right)$, appears. The momentum dependence of these components is shown in Fig. 6(c).

Although $d$-wave superconductivity is stable in a broad range near the AFM critical point, it is significantly suppressed in locally NCS crystals with a large spin-orbit coupling. Thus, we have a chance to see another exotic superconducting state. Candidates are the $B_{1 u}$ and $A_{1 u}$ states which show large eigenvalues of the Eliashberg equation. The other odd-parity IRs are less stable than these states. Both $B_{1 u}$ and $A_{1 u}$ states satisfy the condition $\boldsymbol{d}(\boldsymbol{k}) \| \boldsymbol{g}(\boldsymbol{k})$, in a part of $\boldsymbol{k}$ space, compatible with the selection rule for the intrasublattice pairing. However, $\lambda$ of all the odd-parity IRs moderately decrease as increasing $\alpha$ [inset of Fig. 6(a)] due to the suppression of magnetic fluctuations. Thus, by looking at the $\alpha$ dependence of $\lambda$ we cannot determine which superconducting states are preferred. To examine the superconductivity in a large ASOC region, we calculated the $U$ dependence of $\lambda$ and investigated which superconducting states are stabilized in the vicinity of the magnetic critical point. Figure 6(b) shows that the $B_{1 u}$ state is predominant for $x=0.2$ and the eigenvalue of the Eliashberg equation reaches $\lambda=1$ at $U \simeq 2.4$. The second most stable superconducting state is the $A_{1 u}$ IR. This state is the most stable in the undoped system $x=0$. Thus, odd-parity superconductivity may be realized in a large ASOC region by the magnetic multipole fluctuations.

In order to further elucidate an essential role of sublattice- dependent ASOC, we again compare our model to the model containing a uniform ASOC. Figure 6(e) compares the $U$ dependence of eigenvalues $\lambda$ for the $B_{2 g}$ state and $B_{1 u}$ state. As shown in the lower panel, the $B_{1 u}$ superconducting state is more stable in our model than in the globally NCS model. In other words, the staggered ASOC favors the $B_{1 u}$ state more significantly than does the uniform ASOC. Because the magnetic fluctuation is identified as odd-parity magnetic multipole fluctuation only in the locally NCS model, it is implied that the modification of magnetic fluctuation by the staggered ASOC leads to odd-parity multipole fluctuation and favors odd-parity superconductivity. Note that the $B_{1 u}$ state is compatible with the selection rule in Table IV for both models. In contrast, the even-parity $B_{2 g}$ state is suppressed by the staggered ASOC [upper panel of Fig. 6(e)].

As we show in Table $\mathrm{V}$, the leading order parameter of the $B_{1 u}$ state is an intrasublattice spin-triplet pairing $d^{x 0}(\boldsymbol{k})$ and $d^{y 0}(\boldsymbol{k})$, namely, $\left(\sin k_{x} \bar{\sigma}^{x}-\sin k_{y} \bar{\sigma}^{y}\right) \zeta^{0}$. An admixed staggered spin-singlet component is $d^{0 z}(\boldsymbol{k}) \simeq \delta+\cos k_{x}+\cos k_{y}$. On the other hand, the leading order parameter of the $A_{1 u}$ state is $\left(\sin k_{x} \bar{\sigma}^{x}+\sin k_{y} \bar{\sigma}^{y}\right) \zeta^{0}$, and the induced component is $d^{0 z}(\boldsymbol{k}) \simeq \cos k_{x}-\cos k_{y}$ [see Fig. 6(c)].

Spin-triplet superconductors are known to be a platform of topological superconductivity, which has been one of the main subjects of modern condensed matter physics. The spintriplet superconductivity clarified in this work is also identified as topological superconducting states. According to the criterion for time-reversal-invariant topological superconductivity in two dimension [94], both $B_{1 u}$ and $A_{1 u}$ states are $Z_{2}$ topological superconducting states in class DIII, because the number of Fermi surfaces enclosing time-reversal-invariant momentum ( $\Gamma, X$, and $M$ points) is odd.

Figure 7 shows the phase diagram as a function of $\alpha$ and $U$. From Fig. 7(a) for $x=0.2$, we identify the stable oddparity $B_{1 u}$ state for $\alpha>0.7$, while the $B_{2 g}$ state is stabilized for $\alpha<0.3$. The magnetic instability for $\alpha<0.2$ is the $B_{2 u}$ magnetic quadrupole and hexadecapole order, which is monotonically suppressed by ASOC. The magnetic instability for $\alpha=0.7$ is the $E_{u}$ magnetic quadrupole density wave with an incommensurate period. In an intermediate ASOC region, the $B_{1 g}$ state represented by the predominant intrasublattice spin-singlet pairing $d^{00}(\boldsymbol{k}) \sim \cos k_{x}-\cos k_{y}$ is stable. This state is stabilized by the incommensurate magnetic fluctuation with a small wave vector $\boldsymbol{q} \sim( \pm 1.14, \pm 1.14)$. As is usually done by magnetic fluctuation, the sign change of the gap function between the Fermi surface connected by the wave vector is favored. The $B_{1 g}$ superconducting state is compatible with 
this condition and also with the selection rule for locally NCS superconductors (Table IV).

In the undoped case, $x=0$, we obtain a similar but simpler phase diagram [Fig. 7(b)]. In a large ASOC region, the odd-parity $A_{1 u}$ superconducting state is realized near the ferroic odd-parity magnetic multipole state. The magnetic wave vector is always $\boldsymbol{q}=\mathbf{0}$. Thus, incommensurate magnetic fluctuation is not a necessary condition for odd-parity superconductivity. Irrespective of the wave vector of multipole fluctuations, the odd-parity superconducting states are stabilized in a large ASOC region. In contrast, $B_{1 g}$ superconducting state requires the incommensurate fluctuation, and it disappears in the phase diagram for $x=0$.

Finally, we comment on a peculiar momentum dependence of the gap function protected by nonsymmorphic spacegroup symmetry. From Eq. (19) and a similar equation for $G_{y}=\left\{\sigma_{y} \mid \tau_{x}\right\}$, the intersublattice spin-singlet gap function shows an unusual nodal/gapped structure. As shown in Fig. 8, $d^{0 x}(\boldsymbol{k})$ for the $B_{1 g}$ IR shows nodal lines at $k_{x, y}= \pm \pi$, while it is gapped for the $B_{2 g}$ IR. These nodal/gapped structures at the Brillouin zone boundaries are opposite to those in symmorphic crystals. Note that Fig. 8 does not show fourfold rotation symmetry, because it depicts a real part of the gap function. The superconducting gap is fourfold symmetric in accordance with the symmetry of the system. The gap functions numerically obtained in this paper satisfy the symmetry conditions discussed in Sec. III.

\section{SUMMARY AND DISCUSSION}

In this paper, we have investigated the superconductivity induced by odd-parity magnetic multipole fluctuations in a locally NCS crystal. The obtained results are summarized below.

First, we have revealed the symmetry properties of the superconductivity with sublattice degrees of freedom. The general representation for pair amplitudes including nonsymmorphic operations was derived. After introducing a space group of a specific crystal structure, an unconventional gapped/nodal structure protected by the glide symmetry was shown. For glide-even superconducting states, the intersublattice pair amplitude possesses a node on a Brillouin zone boundary, while those are gapped for glide-odd superconducting states. On the other hand, the intrasublattice pair amplitude shows local parity mixing in a staggered form with respect to the sublattices. The admixture of spin-singlet and spin-triplet pairings is classified by the point group of local atomic sites. These symmetry analyses are consistent with the following numerical results.

Next, we have studied a two-dimensional two-sublattice model with an on-site Coulomb interaction term and a $D_{2 d^{-}}$ type staggered ASOC term. The magnetic fluctuation is suppressed by increasing the ASOC, consistent with a theoretical study of $\mathrm{CePt}_{3} \mathrm{Si}$ [16] and an experiment for $\mathrm{CeCoIn}_{5} \mathrm{su}$ perlattices [92]. The ASOC term also induces an anisotropy in magnetic fluctuation. From the classification of multipole order parameters, the antiferromagnetism of $\boldsymbol{m} \| c$ is clas-
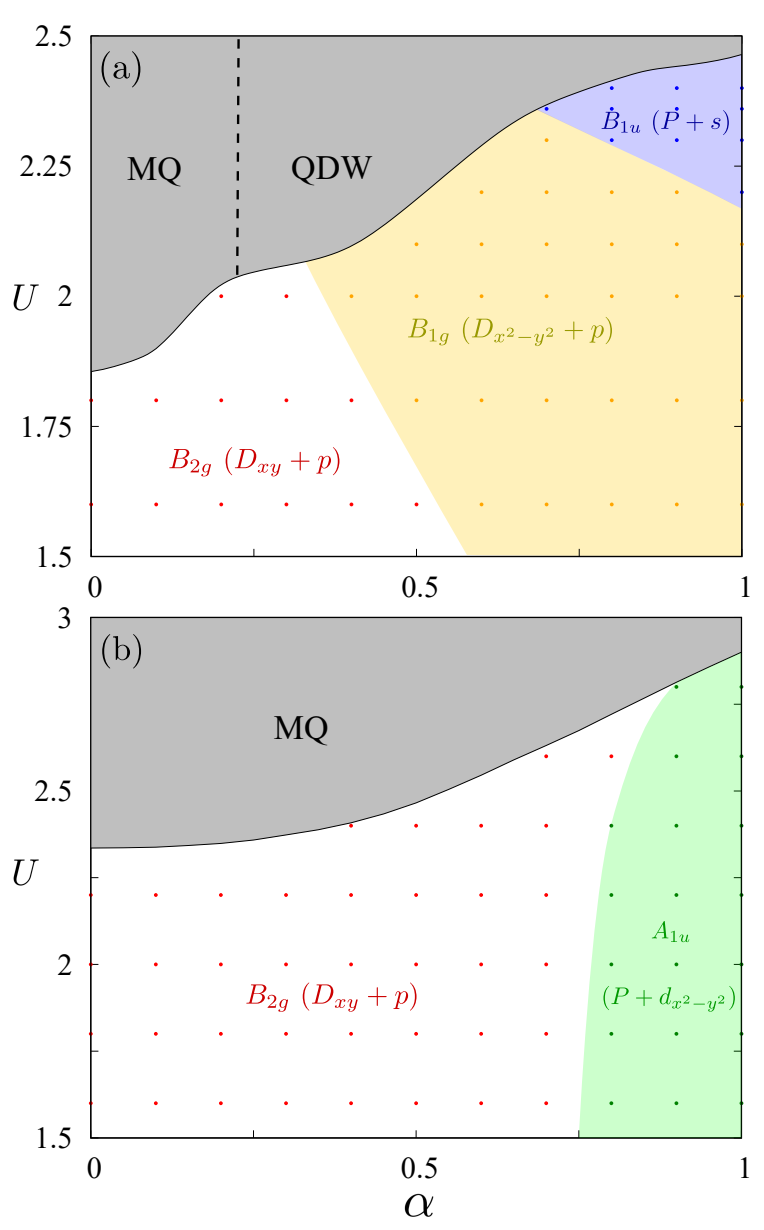

FIG. 7. Phase diagram for the Coulomb interaction $U$ and staggered ASOC $\alpha$ at $T=0.02$. (a) $x=0.2$ and (b) $x=0$. The magnetic quadrupole (MQ) state and quadrupole density wave (QDW) state. In the paramagnetic state, the $B_{2 g}, B_{1 g}, B_{1 u}$, and $A_{1 u}$ superconducting states are illustrated. Capital and lowercase letters represent predominant and parity-mixed (subdominant) components of superconducting order parameter, respectively.

sified into the odd-parity magnetic multipole order belonging to the $B_{2 u}$ IR. In the same way, the $\boldsymbol{m} \perp c$ AFM state is classified into the $E_{u}$ IR. Both $B_{2 u}$ and $E_{u}$ IRs represent odd-parity magnetic multipole orders accompanied by spontaneous global inversion symmetry breaking. In our model, these odd-parity multipole fluctuations are enhanced in the vicinity of the magnetic critical point.

Superconducting instability has been analyzed by solving the Eliashberg equation with the use of RPA. We have demonstrated the selection rules of locally NCS superconductors [3]. Since the intersublattice spin-singlet pairing is ruled out by the selection rule, the $B_{2 g}$ state, which corresponds to the well-studied $d_{x^{2}-y^{2}}$-wave superconducting state in the singlesublattice Hubbard model, is rapidly suppressed by turning on the ASOC. Intriguingly, this behavior is in sharp contrast with globally NCS superconductors. When $d$-wave superconductivity is suppressed in a large ASOC region, the odd-parity 

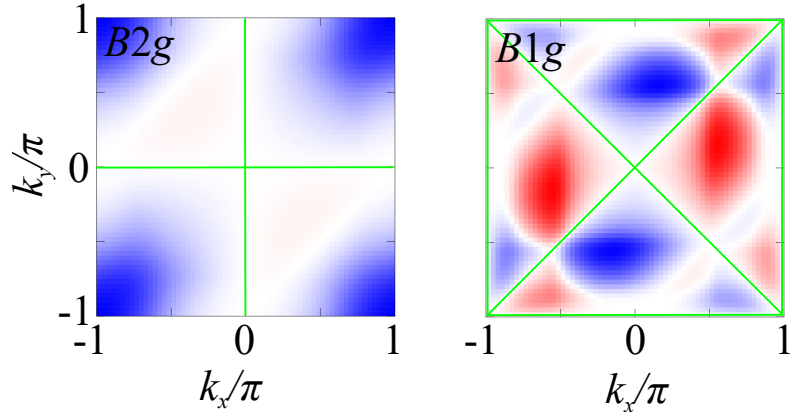

FIG. 8. Intersublattice components of the gap function for $U=1.4$ and $\alpha=0.1$. We show the real part for (a) $B_{2 g}$ IR and (b) $B_{1 g}$ IR.

superconductivity is stabilized by enhanced odd-parity multipole fluctuations. We found that the $B_{1 u}$ or $A_{1 u}$ state is stable. From the criterion for time-reversal-invariant topological superconductivity, both the $B_{1 u}$ and $A_{1 u}$ states are identified as the nontrivial $Z_{2}$ topological superconductivity in the DIII class. Thus, our results may open a different platform of oddparity topological superconductivity.

Here, we note that the staggered ASOC arising from the local parity violation in the crystal structure plays an essential role in stabilizing odd-parity superconductivity. From a comparison with the globally NCS model, we have shown that the modification of magnetic fluctuation by the staggered ASOC significantly enhances odd-parity superconductivity. Such an enhancement is not caused by the sublattice-independent ASOC in the globally NCS system. The modified magnetic fluctuation in the locally NCS system is regarded as odd-parity magnetic multipole fluctuations. Therefore, we conclude that odd-parity superconductivity is stabilized by odd-parity multipole fluctuations.

In the long-standing studies of unconventional superconductivity, spin-triplet superconductivity has attracted interest. Furthermore, renewed interest has been stimulated because odd-parity superconductivity may be topologically nontrivial. However, only limited examples, such as $\mathrm{Sr}_{2} \mathrm{RuO}_{4}$ [95], $\mathrm{UPt}_{3}$ [96], UCoGe [97], and so on, are known as strong candidates for spin-triplet superconductors. This is because conditions for spin-triplet pairing are quite unfavorable in most materials. Our paper has uncovered a different pairing mechanism favorable for spin-triplet pairing. The local parity violation in crystal structures, large spin-orbit coupling, and enhanced magnetic multipole fluctuations are conditions for spin-triplet superconductivity proposed in this paper.

Finally, we discuss candidate materials of odd-parity fluctuation and superconductivity. First, $\mathrm{Sr}_{2} \mathrm{IrO}_{4}$ is a layered perovskite $5 d$ transition metal oxide and possesses a $\mathrm{K}_{2} \mathrm{NiF}_{4}$-type structure as does $\mathrm{La}_{2} \mathrm{CuO}_{4}$. Many similarities to the high- temperature cuprate superconductors have been recognized, and thus it is expected to be a superconductor from both the experimental [54] and theoretical [39, 60] sides. AFM moments align to the $a$ axis with a small canted moment along the $b$ axis and show stacking patterns:,-++--+-+ , and ++++ . From the viewpoint of multipole order, the -++pattern is magnetic octupole order preserving space inversion symmetry, while the -+-+ pattern is odd-parity magnetic quadrupole order [40]. Another candidate is $\mathrm{BaMn}_{2} \mathrm{As}_{2}$ crystallizing in a locally NCS $\mathrm{ThCr}_{2} \mathrm{Si}_{2}$-type structure, which is isostructural to the 122 systems of iron-based superconductors. Undoped $\mathrm{BaMn}_{2} \mathrm{As}_{2}$ shows the $G$-type AFM order at $T_{N}=625 \mathrm{~K}$ [48]. The magnetic structure is classified into the odd-parity magnetic quadrupole and hexadecapole orders [28]. Many related materials show the same odd-parity magnetic order, and some of them may be superconducting [75]. A further experimental search is desired. Hole-doped $\left(\mathrm{Ba}_{1-x} \mathrm{~K}_{x}\right) \mathrm{Mn}_{2} \mathrm{As}_{2}$ realizes the metallic state. However, superconductivity has not been observed up to now. A fascinating material is CrAs [98-106]. The space group is No. 62, Pnma ( $D_{2 h}$ point group) lacking local inversion symmetry at the $\mathrm{Cr}$ sites. CrAs shows a first-order helical magnetic transition at $T_{N} \sim 265 \mathrm{~K}[98,99]$. When the helical magnetic order is suppressed by applied pressure, superconductivity occurs [100]. The phase diagram implies superconductivity induced by magnetic fluctuation. The wave vector of the helical magnetism is incommensurate, $\boldsymbol{q}=\left(0,0, q_{c}\right)$ with $q_{c} \sim 0.354$. Thus, a local parity violation and odd-parity magnetic fluctuation may promote odd-parity superconductivity in CrAs. Indeed, a recent experiment suggests spin-triplet superconductivity [106]. As for odd-parity electric multipole fluctuation, $\mathrm{SrTiO}_{3}$ [74] and $\mathrm{Cd}_{2} \mathrm{Re}_{2} \mathrm{O}_{7}$ [67] show superconductivity in the vicinity of the nonmagnetic order accompanied by global inversion symmetry breaking. Recently, Ref. 76 theoretically proposed that odd-parity electric fluctuation may induce the odd-parity superconductivity. More research of multipole materials will shed light on odd-parity superconductivity in condensed matter.

\section{ACKNOWLEDGMENTS}

The authors are grateful to S. Sumita, H. Watanabe, Y. Yanagi, and A. Daido for fruitful discussions and comments. This work was supported by a Grant-in-Aid for Scientific Research on Innovative Areas "J-Physics" (Grant No. JP15H05884) and "Topological Materials Science" (Grants No. JP16H00991 and JP18H04225) from Japan Society for Promotion of Science (JSPS) and by JSPS KAKENHI (Grants No. JP15K05164, No. JP15H05745, and No. JP18H01178).
[1] M. Sigrist, D. F. Agterberg, M. H. Fischer, J. Goryo, F. Loder, S.-H. Rhim, D. Maruyama, Y. Yanase, T. Yoshida, and S. J.
Youn, J. Phys. Soc. Jpn. 83, 061014 (2014). 
[2] D. Maruyama, M. Sigrist, and Y. Yanase, J. Phys. Soc. Jpn. 81, 034702 (2012).

[3] M. H. Fischer, F. Loder, and M. Sigrist, Phys. Rev. B 84, 184533 (2011).

[4] S. Nakosai, Y. Tanaka, and N. Nagaosa, Phys. Rev. Lett. 108, 147003 (2012).

[5] T. Yoshida, M. Sigrist, and Y. Yanase, Phys. Rev. B 86, 134514 (2012).

[6] T. Yoshida, M. Sigrist, and Y. Yanase, J. Phys. Soc. Jpn. 82, 074714 (2013).

[7] T. Yoshida, M. Sigrist, and Y. Yanase, Phys. Rev. Lett. 115, 027001 (2015).

[8] T. Yoshida, A. Daido, Y. Yanase, and N. Kawakami, Phys. Rev. Lett. 118, 147001 (2017).

[9] Non-Centrosymmetric Superconductors: Introduction and Overview, edited by E. Bauer and M. Sigrist, Lecture Notes in Physics Vol. 847 (Springer, Berlin, 2012).

[10] R. Settai, T. Takeuchi, and Y. Ōnuki, J. Phys. Soc. Jpn. 76, 051003 (2007).

[11] V. M. Edelstein, Phys. Rev. Lett. 75, 2004 (1995).

[12] L. P. Gor'kov and E. I. Rashba, Phys. Rev. Lett. 87, 037004 (2001).

[13] P. A. Frigeri, D. F. Agterberg, A. Koga, and M. Sigrist, Phys. Rev. Lett. 92, 097001 (2004).

[14] K. V. Samokhin, Phys. Rev. B 70, 104521 (2004).

[15] Y. Yanase and M. Sigrist, J. Phys. Soc. Jpn. 76, 043712 (2007).

[16] Y. Yanase and M. Sigrist, J. Phys. Soc. Jpn. 77, 124711 (2008).

[17] T. Yokoyama, S. Onari, and Y. Tanaka, Phys. Rev. B 75, 172511 (2007).

[18] Y. Tada, N. Kawakami, and S. Fujimoto, New Journal of Physics 11, 055070 (2009).

[19] D. F. Agterberg and R. P. Kaur, Phys. Rev. B 75, 064511 (2007).

[20] S. Fujimoto, J. Phys. Soc. Jpn. 75, 083704 (2006).

[21] S. Fujimoto, J. Phys. Soc. Jpn. 76, 034712 (2007).

[22] P. M. R. Brydon, L. Wang, M. Weinert, and D. F. Agterberg, Phys. Rev. Lett. 116, 177001 (2016).

[23] E. Bauer, G. Hilscher, H. Michor, C. Paul, E. W. Scheidt, A. Gribanov, Y. Seropegin, H. Noël, M. Sigrist, and P. Rogl, Phys. Rev. Lett. 92, 027003 (2004).

[24] I. Sugitani, Y. Okuda, H. Shishido, T. Yamada, A. Thamizhavel, E. Yamamoto, T. D. Matsuda, Y. Haga, T. Takeuchi, R. Settai, and Y. Ōnuki, J. Phys. Soc. Jpn. 75, 043703 (2006).

[25] Y. Yanase, J. Phys. Soc. Jpn. 83, 014703 (2014).

[26] J. Železný, H. Gao, K. Výborný, J. Zemen, J. Mašek, A. Manchon, J. Wunderlich, J. Sinova, and T. Jungwirth, Phys. Rev. Lett. 113, 157201 (2014).

[27] P. Wadley, B. Howells, J. Železný, C. Andrews, V. Hills, R. P. Campion, V. Novák, K. Olejník, F. Maccherozzi, S. S. Dhesi, S. Y. Martin, T. Wagner, J. Wunderlich, F. Freimuth, Y. Mokrousov, J. Kuneš, J. S. Chauhan, M. J. Grzybowski, A. W. Rushforth, K. W. Edmonds, B. L. Gallagher, and T. Jungwirth, Science 351, 587 (2016).

[28] H. Watanabe and Y. Yanase, Phys. Rev. B 96, 064432 (2017).

[29] K. Kuroki, S. Onari, R. Arita, H. Usui, Y. Tanaka, H. Kontani, and H. Aoki, Phys. Rev. Lett. 101, 087004 (2008).

[30] Y. Kamihara, T. Watanabe, M. Hirano, and H. Hosono, J. Am. Chem. Soc. 130, 3296 (2008).

[31] Y. Mizukami, H. Shishido, T. Shibauchi, M. Shimozawa, S. Yasumoto, D. Watanabe, M. Yamashita, H. Ikeda, T. Terashima, H. Kontani, and Y. Matsuda, Nat. Phys. 7, 849 (2011).

[32] S. Sumita and Y. Yanase, Phys. Rev. B 93, 224507 (2016).
[33] L. Fu, Phys. Rev. Lett. 115, 026401 (2015).

[34] T. Hitomi and Y. Yanase, J. Phys. Soc. Jpn. 83, 114704 (2014).

[35] T. Hitomi and Y. Yanase, J. Phys. Soc. Jpn. 85, 124702 (2016).

[36] S. Hayami, H. Kusunose, and Y. Motome, Phys. Rev. B 90, 024432 (2014).

[37] S. Hayami, H. Kusunose, and Y. Motome, Phys. Rev. B 90, 081115 (2014)

[38] S. Hayami, H. Kusunose, and Y. Motome, J. Phys. Soc. Jpn. 84, 064717 (2015).

[39] S. Sumita, T. Nomoto, and Y. Yanase, Phys. Rev. Lett. 119, 027001 (2017).

[40] S. Di Matteo and M. R. Norman, Phys. Rev. B 94, 075148 (2016).

[41] S. Hayami, H. Kusunose, and Y. Motome, Phys. Rev. B 97, 024414 (2018).

[42] Y. Yanagi, S. Hayami, and H. Kusunose, Phys. Rev. B 97, 020404 (2018).

[43] S. Hayami and H. Kusunose, J. Phys. Soc. Jpn. 87, 033709 (2018).

[44] Y. Kuramoto, H. Kusunose, and A. Kiss, J. Phys. Soc. Jpn. 78, 072001 (2009).

[45] K. Haule and G. Kotliar, Nat. Phys. 5, 796 (2009).

[46] H. Kusunose and H. Harima, J. Phys. Soc. Jpn. 80, 084702 (2011).

[47] H. Ikeda, M.-T. Suzuki, R. Arita, T. Takimoto, T. Shibauchi, and Y. Matsuda, Nat. Phys. 8, 528 (2012).

[48] Y. Singh, M. A. Green, Q. Huang, A. Kreyssig, R. J. McQueeney, D. C. Johnston, and A. I. Goldman, Phys. Rev. B 80, 100403 (2009).

[49] L. Zhao, D. H. Torchinsky, H. Chu, V. Ivanov, R. Lifshitz, R. Flint, T. Qi, G. Cao, and D. Hsieh, Nat. Phys. 12, 32 (2015).

[50] B. J. Kim, H. Jin, S. J. Moon, J.-Y. Kim, B.-G. Park, C. S. Leem, J. Yu, T. W. Noh, C. Kim, S.-J. Oh, J.-H. Park, V. Durairaj, G. Cao, and E. Rotenberg, Phys. Rev. Lett. 101, 076402 (2008).

[51] Y. K. Kim, O. Krupin, J. D. Denlinger, A. Bostwick, E. Rotenberg, Q. Zhao, J. F. Mitchell, J. W. Allen, and B. J. Kim, Science 345, 187 (2014).

[52] Y. J. Yan, M. Q. Ren, H. C. Xu, B. P. Xie, R. Tao, H. Y. Choi, N. Lee, Y. J. Choi, T. Zhang, and D. L. Feng, Phys. Rev. X 5, 041018 (2015)

[53] I. Battisti, K. M. Bastiaans, V. Fedoseev, A. de la Torre, N. Iliopoulos, A. Tamai, E. C. Hunter, R. S. Perry, J. Zaanen, F. Baumberger, and M. P. Allan, Nat. Phys. 13, 21 (2016).

[54] Y. K. Kim, N. H. Sung, J. D. Denlinger, and B. J. Kim, Nat. Phys. 12, 37 (2016).

[55] Z. Y. Meng, Y. B. Kim, and H.-Y. Kee, Phys. Rev. Lett. 113, 177003 (2014).

[56] B. J. Kim, H. Ohsumi, T. Komesu, S. Sakai, T. Morita, H. Takagi, and T. Arima, Science 323, 1329 (2009).

[57] S. Boseggia, H. C. Walker, J. Vale, R. Springell, Z. Feng, R. S. Perry, M. M. Sala, H. M. Rønnow, S. P. Collins, and D. F. McMorrow, J. Phys.: Condens. Matter 25, 422202 (2013).

[58] J. P. Clancy, A. Lupascu, H. Gretarsson, Z. Islam, Y. F. Hu, D. Casa, C. S. Nelson, S. C. LaMarra, G. Cao, and Y.-J. Kim, Phys. Rev. B 89, 054409 (2014).

[59] C. Dhital, T. Hogan, Z. Yamani, C. de la Cruz, X. Chen, S. Khadka, Z. Ren, and S. D. Wilson, Phys. Rev. B 87, 144405 (2013).

[60] H. Watanabe, T. Shirakawa, and S. Yunoki, Phys. Rev. Lett. 110, 027002 (2013).

[61] Q. Huang, J. Soubeyroux, O. Chmaissem, I. Sora, A. Santoro, R. Cava, J. Krajewski, and W. Peck, J. Solid State Chem. 112, 355 (1994). 
[62] M. K. Crawford, M. A. Subramanian, R. L. Harlow, J. A. Fernandez-Baca, Z. R. Wang, and D. C. Johnston, Phys. Rev. B 49, 9198 (1994).

[63] J. S. Dodge, Science 356, 246 (2017).

[64] J. W. Harter, Z. Y. Zhao, J.-Q. Yan, D. G. Mandrus, and D. Hsieh, Science 356, 295 (2017).

[65] S. Di Matteo and M. R. Norman, Phys. Rev. B 96, 115156 (2017).

[66] H. Harima, Journal of Physics and Chemistry of Solids 63, 1035 (2002), proceedings of the 8th ISSP International Symposium.

[67] J.-i. Yamaura, K. Takeda, Y. Ikeda, N. Hirao, Y. Ohishi, T. C. Kobayashi, and Z. Hiroi, Phys. Rev. B 95, 020102 (2017).

[68] J.-I. Yamaura and Z. Hiroi, J. Phys. Soc. Jpn. 71, 2598 (2002).

[69] Z. Hiroi, J.-I. Yamaura, Y. Muraoka, and M. Hanawa, J. Phys. Soc. Jpn. 71, 1634 (2002).

[70] M. Hanawa, J. Yamaura, Y. Muraoka, F. Sakai, and Z. Hiroi, Journal of Physics and Chemistry of Solids 63, 1027 (2002), proceedings of the 8th ISSP International Symposium.

[71] H. Sakai, K. Yoshimura, H. Ohno, H. Kato, S. Kambe, R. E. Walstedt, T. D. Matsuda, Y. Haga, and Y. Ōnuki, J. Phys.: Condens. Matter 13, L785 (2001).

[72] J. G. Bednorz and K. A. Müller, Phys. Rev. Lett. 52, 2289 (1984).

[73] J. F. Schooley, W. R. Hosler, and M. L. Cohen, Phys. Rev. Lett. 12, 474 (1964).

[74] C. W. Rischau, X. Lin, C. P. Grams, D. Finck, S. Harms, J. Engelmayer, T. Lorenz, Y. Gallais, B. Fauqué, J. Hemberger, and K. Behnia, Nat. Phys. 13, 643 (2017).

[75] H. Watanabe and Y. Yanase, ArXiv e-prints , arXiv:1805.10828.

[76] V. Kozii and L. Fu, Phys. Rev. Lett. 115, 207002 (2015).

[77] Y. Wang, G. Y. Cho, T. L. Hughes, and E. Fradkin, Phys. Rev. B 93, 134512 (2016).

[78] J. M. Edge, Y. Kedem, U. Aschauer, N. A. Spaldin, and A. V. Balatsky, Phys. Rev. Lett. 115, 247002 (2015).

[79] T. Takimoto and P. Thalmeier, J. Phys. Soc. Jpn. 78, 103703 (2009).

[80] M. Sigrist and K. Ueda, Rev. Mod. Phys. 63, 239 (1991).

[81] T. Nomoto, K. Hattori, and H. Ikeda, Phys. Rev. B 94, 174513 (2016).

[82] R. Heid, Z. Phys. B: Condens. Matter 99, 15 (1995).

[83] Y. Yanase, Phys. Rev. B 94, 174502 (2016).

[84] T. Nomoto and H. Ikeda, Phys. Rev. Lett. 117, 217002 (2016).

[85] T. Nomoto and H. Ikeda, J. Phys. Soc. Jpn. 86, 023703 (2017).

[86] M. R. Norman, Phys. Rev. B 52, 15093 (1995).

[87] T. Micklitz and M. R. Norman, Phys. Rev. B 80, 100506 (2009).

[88] S. Kobayashi, Y. Yanase, and M. Sato, Phys. Rev. B 94, 134512 (2016).
[89] S. Sumita and Y. Yanase, Phys. Rev. B 97, 134512 (2018).

[90] S. Kobayashi, S. Sumita, Y. Yanase, and M. Sato, Phys. Rev. B 97, 180504 (2018).

[91] Y. Yanase, T. Jujo, T. Nomura, H. Ikeda, T. Hotta, and K. Yamada, Phys. Rep. 387, 1 (2003).

[92] T. Yamanaka, M. Shimozawa, R. Endo, Y. Mizukami, H. Shishido, T. Terashima, T. Shibauchi, Y. Matsuda, and K. Ishida, Phys. Rev. B 92, 241105 (2015).

[93] Because nonsymmorphic space groups contain nonprimitive translation operations, there are several choices of an inversion center, giving an ambiguity of an atomic cluster. In the present case, we have two choices for the unit cell. One is shown in Fig. 1, and the other is obtained by the glide operation. $\chi_{B_{2 u}\left(E_{u}\right)}(\boldsymbol{q})$ at $\boldsymbol{q} \neq(0,0)$ depends on the choice of the unit cell. To avoid this ambiguity, we define bulk susceptibility by average over the choices of the unit cell, $\chi_{B_{2 u}\left(E_{u}\right)}(\boldsymbol{q})=$ $\left(\chi_{B_{2 u}\left(E_{u}\right)}^{(1)}(\boldsymbol{q})+\chi_{B_{2 u}\left(E_{u}\right)}^{(2)}(\boldsymbol{q})\right) / 2$.

[94] M. Sato, Phys. Rev. B 81, 220504 (2010).

[95] A. P. Mackenzie and Y. Maeno, Rev. Mod. Phys. 75, 657 (2003).

[96] R. Joynt and L. Taillefer, Rev. Mod. Phys. 74, 235 (2002).

[97] N. T. Huy, A. Gasparini, D. E. de Nijs, Y. Huang, J. C. P. Klaasse, T. Gortenmulder, A. de Visser, A. Hamann, T. Görlach, and H. v. Löhneysen, Phys. Rev. Lett. 99, 067006 (2007).

[98] H. Watanabe, N. Kazama, Y. Yamaguchi, and M. Ohashi, J. Appl. Phys. 40, 1128 (1969).

[99] K. Selte, A. Kjekshus, W. E. Jamison, A. Andresen, and J. E. Engebretsen, Acta Chem. Scand. 25, 1703 (1971).

[100] H. Kotegawa, S. Nakahara, H. Tou, and H. Sugawara, J. Phys. Soc. Jpn. 83, 093702 (2014).

[101] W. Wu, J. Cheng, K. Matsubayashi, P. Kong, F. Lin, C. Jin, N. Wang, Y. Uwatoko, and J. Luo, Nat. Commun. 5, 5508 (2014).

[102] H. Kotegawa, S. Nakahara, R. Akamatsu, H. Tou, H. Sugawara, and H. Harima, Phys. Rev. Lett. 114, 117002 (2015).

[103] L. Keller, J. S. White, M. Frontzek, P. Babkevich, M. A. Susner, Z. C. Sims, A. S. Sefat, H. M. Rønnow, and C. Rüegg, Phys. Rev. B 91, 020409 (2015).

[104] Y. Shen, Q. Wang, Y. Hao, B. Pan, Y. Feng, Q. Huang, L. W. Harriger, J. B. Leao, Y. Zhao, R. M. Chisnell, J. W. Lynn, H. Cao, J. Hu, and J. Zhao, Phys. Rev. B 93, 060503 (2016).

[105] Q. Niu, W. C. Yu, K. Y. Yip, Z. L. Lim, H. Kotegawa, E. Matsuoka, H. Sugawara, H. Tou, Y. Yanase, and S. K. Goh, Nat. Commun. 8, 15358 (2017).

[106] C. Y. Guo, M. Smidman, B. Shen, W. Wu, F. K. Lin, X. L. Han, Y. Chen, F. Wu, Y. F. Wang, W. B. Jiang, X. Lu, J. P. Hu, J. L. Luo, and H. Q. Yuan, Phys. Rev. B 98, 024520 (2018). 